\title{
Marine Natural Products from Tunicates and Their Associated Microbes
}

\author{
Chatragadda Ramesh ${ }^{1,2, *}$, Bhushan Rao Tulasi ${ }^{3}$, Mohanraju Raju ${ }^{2}$, Narsinh Thakur ${ }^{4}$ and Laurent Dufossé ${ }^{5, *(D)}$ \\ 1 Biological Oceanography Division (BOD), CSIR-National Institute of Oceanography (CSIR-NIO), \\ Dona Paula 403004, India \\ 2 Department of Ocean Studies and Marine Biology, Pondicherry Central University, Brookshabad Campus, \\ Port Blair 744102, India; mohanrajupu62@gmail.com \\ 3 Zoology Division, Sri Gurajada Appa Rao Government Degree College, Yellamanchili 531055, India; \\ bhushanphd@gmail.com \\ 4 Chemical Oceanography Division (COD), CSIR-National Institute of Oceanography (CSIR-NIO), \\ Dona Paula 403004, India; thakurn@nio.org \\ 5 Laboratoire de Chimie et Biotechnologie des Produits Naturels (CHEMBIOPRO), Université de La Réunion, \\ ESIROI Agroalimentaire, 15 Avenue René Cassin, CS 92003, CEDEX 9, \\ F-97744 Saint-Denis, Ile de La Réunion, France \\ * Correspondence: chramesh@nio.org (C.R.); laurent.dufosse@univ-reunion.fr (L.D.); \\ Tel.: +91-(0)-832-2450636 (C.R.); +33-668-731-906 (L.D.)
}

Citation: Ramesh, C.; Tulasi, B.R.; Raju, M.; Thakur, N.; Dufossé, L. Marine Natural Products from Tunicates and Their Associated Microbes. Mar. Drugs 2021, 19, 308. https://doi.org/10.3390/md19060308

Academic Editors: Concetta Imperatore, Marialuisa Menna and Marcello Casertano

Received: 4 May 2021

Accepted: 24 May 2021

Published: 26 May 2021

Publisher's Note: MDPI stays neutral with regard to jurisdictional claims in published maps and institutional affiliations.

Copyright: (c) 2021 by the authors. Licensee MDPI, Basel, Switzerland. This article is an open access article distributed under the terms and conditions of the Creative Commons Attribution (CC BY) license (https:// creativecommons.org/licenses/by/ $4.0 /$ )

\begin{abstract}
Marine tunicates are identified as a potential source of marine natural products (MNPs) demonstrating a wide range of biological properties, like antimicrobial and anticancer activities. The symbiotic relationship between tunicates and specific microbial groups has revealed the acquisition of microbial compounds by tunicates for defensive purpose. For instance, yellow pigmented compounds, "tambjamines", produced by the tunicate, Sigillina signifera (Sluiter, 1909), primarily originated from their bacterial symbionts, which are involved in their chemical defense function, indicating the ecological role of symbiotic microbial association with tunicates. This review has garnered comprehensive literature on MNPs produced by tunicates and their symbiotic microbionts. Various sections covered in this review include tunicates' ecological functions, biological activities, such as antimicrobial, antitumor, and anticancer activities, metabolic origins, utilization of invasive tunicates, and research gaps. Apart from the literature content, 20 different chemical databases were explored to identify tunicates-derived MNPs. In addition, the management and exploitation of tunicate resources in the global oceans are detailed for their ecological and biotechnological implications.
\end{abstract}

Keywords: tunicates; symbiotic microbes; pigments; bioactive compounds; alkaloids \&amp; peptides

\section{Introduction}

Tunicates and sea squirts are soft-bodied solitary or colonial (60\%) sessile marine organisms belonging to the family Ascidiacea under the subphylum Urochordata, phylum Chordata [1,2]. These organisms are hermaphroditic, filter feeders, and appear in different body colors, such as translucent to blue, green, yellow, red, and brown, with a life span ranging from two months to one year [1-4]. Currently, tunicates are classified into four major clades such as (a) Appendicularia, (b) Thaliacea + Phlebobranchia + Aplousobranchia, (c) Molgulidae, and (d) Styelidae + Pyuridae, on the basis of the phylogenomic transcriptomic approach [5]. Globally, around 2815 tunicate species have been recorded from shallow coastal waters to deep waters [1]. Tunicate larvae resemble tadpole larvae of members of Chordata, but soon after the retrogressive metamorphosis, they lose the notochord and post-anal tail; thus, these organisms are often referred to as the "evolutionary connecting link" between invertebrates and chordates [6,7]. Therefore, tunicates are considered as important model organisms for several research aspects, such as evolution [6], development biology [8,9], invasion success [10], and bioactive compounds. 
Tunicates are prolific producers of marine natural products (MNPs), and certain species are also known to release toxins, such as Bistramide A [11,12]. However, a few species, like Halocynthia roretzi and Pyura michaelseni, are eaten in southeast Asian countries like Korea $[13,14]$. The strong immune defensive system [15] and their associated symbiotic microbes with bioactive properties [16], makes tunicates highly preferential drug resources in the ocean [15,17]. Since the majority of the tunicate species are known to produce MNP's, attempts are being undertaken in the culturing of these tunicates (e.g., mangrove tunicate Ecteinascidia turbinata) in large scale for various applications $[18,19]$. The process of accumulation of vanadium by vanadocytes of tunicates from seawater is well-known [20]. In contrast, investigations on the acquisition of MNPs by tunicates from their symbiotic bacteria are very limited, except for the antitumor products ecteinascidins [21,22], didemnin [23], and talaropeptides [24]. A recent review highlighted the association of bacteria, actinomycetes, fungi, and cyanobacteria with the tunicates and their bioactive nature [25]. It was also observed that actinomycetes, fungi, and bacteria are the predominant microbes associated with the tunicates, showing cytotoxic and antimicrobial activities [26], with the production of alkaloids as the major source of MNPs [27]. In this context, this review aimed to provide the chemical profiles of various tunicates and their associated microbes for biotechnological and drug development applications.

\section{Ecological Importance of Tunicates}

The tunicates population plays an important role in the marine food web through filter feeding [4]. Earlier studies have suggested that phytoplankton productivity in a shallow fjord is controlled by the tunicates population [28]. Tunicates are known to trap the sinking particulate organic matter and generate mucus rich organic matter and fecal pellets with carbohydrates and minerals $[29,30]$, thereby triggering the downward biogeochemical flux (e.g., carbon flux) patterns from surface to deep waters [29,31,32]. Some obligate photosymbiotic tunicates have been suggested to act as environmental stress indicators [33]. The unknown ecological functions of a few tunicate MNPs [34] in understanding their ecological role is yet to be understood.

\section{Database Search on Tunicate MNPs}

Twenty different public chemical databases such as BIAdb, BindingDB, ChemDB, ChEMBL, ChemSpider, DrugBank, HIT, HMDB, KEGG, NCI, NPACT, PDB-Bind, PDBeChem, PharmaGKB, PubChem, SMPDB, SuperDrug, TTD, UNIProt, and ZINC were explored to identify the tunicate-originated MNPs deposited in these databases. The chemical constituents identified from these databases using the search keywords "tunicate and ascidian" are listed in Table 1.

Table 1. List of MNPs originated from tunicates available in various public databases. The unknown compound records are excluded from the list.

\begin{tabular}{ccccc}
\hline Database & $\begin{array}{c}\text { No. of Known } \\
\text { Compounds }\end{array}$ & $\begin{array}{c}\text { No. of Unknown } \\
\text { Compounds }\end{array}$ & $\begin{array}{c}\text { Known Chemical } \\
\text { Compound }\end{array}$ & Biological Properties \\
\hline BIAdb & 1 & - & Polycarpine & $\begin{array}{c}\text { Cytotoxic, antiviral, and } \\
\text { antifungal }\end{array}$ \\
\hline BindingDB & 2 & - & $\begin{array}{c}\text { Tuberatolides, } \\
\text { Sodium 1-(12-hydroxy) } \\
\text { octadecanyl sulfate }\end{array}$ & $\begin{array}{c}\text { Farnesoid X receptor } \\
\text { antagonists, matrix } \\
\text { metalloproteinase 2 inhibitor }\end{array}$ \\
\hline ChemDB & 2 & $\begin{array}{c}\text { Patellazole B, } \\
\text { Patellazole C }\end{array}$ & Antimicrobial, cytotoxic \\
\hline ChEMBL & 2 & Ascididemin, & Anticancer \\
\hline ChemSpider & 1 & - & Trabectedin & Anticancer \\
\hline
\end{tabular}


Table 1. Cont.

\begin{tabular}{|c|c|c|c|c|}
\hline Database & $\begin{array}{l}\text { No. of Known } \\
\text { Compounds }\end{array}$ & $\begin{array}{l}\text { No. of Unknown } \\
\text { Compounds }\end{array}$ & $\begin{array}{c}\text { Known Chemical } \\
\text { Compound }\end{array}$ & Biological Properties \\
\hline DrugBank & - & - & & \\
\hline HIT & - & - & & \\
\hline HMDB & 1 & - & Trabectedin & Anticancer \\
\hline KEGG & 1 & - & Trabectedin & Anticancer \\
\hline $\mathrm{NCI}$ & - & - & & \\
\hline NPACT & - & - & & \\
\hline PDB-Bind & - & - & & \\
\hline PDBeChem & 16 & $>30$ & $\begin{array}{c}\text { Cystodytin D, cystodytin F, } \\
\text { cystodytin E, } \\
\text { cystodytin } \mathrm{G}, \\
\text { cystodytin } \mathrm{H}, \\
\text { cystodytin I, } \\
\text { Diplosoma ylidene 1, } \\
\text { Diplosoma ylidene 2, } \\
\text { Lejimalide A, lejimalide B, } \\
\text { lissoclibadin 1, } \\
\text { lissoclibadin 2, } \\
\text { lissoclibadin 3, lamellarin } \\
\text { alpha 20-sulfate, } \\
\text { plitidepsin, } \\
\text { trabectedin }\end{array}$ & Cytotoxic, anticancer \\
\hline PharmaGKB & 1 & - & Trabectedin & Anticancer \\
\hline PubChem & 4 & 2 & $\begin{array}{c}\text { Patellazole B, } \\
\text { Patellazole C, } \\
\text { GnRH-II, } \\
\text { GnRH-I }\end{array}$ & $\begin{array}{l}\text { Antimicrobial and cytotoxic, } \\
\text { induces spawning }\end{array}$ \\
\hline SMPDB & - & - & & \\
\hline SuperDrug & 1 & - & Trabectedin & Anticancer \\
\hline TTD & - & - & & \\
\hline UniProt & 1 & 1 & Retinoic acid & Regeneration of gut \\
\hline ZINC & 1 & - & Trabectedin & Anticancer \\
\hline
\end{tabular}

Foot note: Table 1 data are garnered from public chemical databases listed in the main text part 3 , but not from the literature. That is why there are no references cited in this table. Readers are asked to refer to Tables 2 and 3 where details are from the literature, and therefore, references are cited.

\section{Profile of MNPs from Tunicates and Associated Microbes}

Tunicates are known to produce a wide range of MNPs with various bioactive properties (Tables 2 and 3). These organisms are considered as a rich source of cellulose, which varies with different species [35]. Alkaloids and peptides are the major chemical constituents observed in tunicates [36]. Metabolites originated from tunicate hemocytes are also found to be cytotoxic to foreign particles [37] and various cell lines [38]. Microorganisms associated with the invertebrate hosts have also been identified as a source of bioactive metabolites [39]. In fact, bioactive metabolite-producing invertebrate-associated microorganisms have special implications in solving the "supply problem" in the initial steps of drug discovery [40]. Recently, Chen et al. reviewed the biological and chemical diversity of ascidian-associated microorganisms [41]. 
Table 2. Chemical profiles from body parts and fluids of few tunicate species.

\begin{tabular}{|c|c|c|c|c|}
\hline Body Component & Chemical Compound & Function & Application & Reference \\
\hline $\begin{array}{c}\text { Tunic } \\
\text { (Ascidia sp., Ciona intestinalis, } \\
\text { Halocynthia roretzi, and Styela plicata) }\end{array}$ & Tunicin (cellulose) & Protection & Material cellulose & {$[35]$} \\
\hline $\begin{array}{l}\text { Blood (Ascidia nigra, } \\
\text { Molgula manhattensis) }\end{array}$ & Vanadium & Oxygen transport & & {$[42]$} \\
\hline $\begin{array}{c}\text { Blood } \\
\text { (Ascidia nigra) }\end{array}$ & Tunichromes & $\begin{array}{l}\text { Vanadium binding and } \\
\text { reduces blood pigments }\end{array}$ & & {$[42,43]$} \\
\hline $\begin{array}{l}\text { Hemocytes } \\
(\text { Styela clava) }\end{array}$ & Clavanins & Multiple functions & Antimicrobial & {$[44,45]$} \\
\hline $\begin{array}{c}\text { Hemocytes } \\
\text { (Halocynthia papillosa) }\end{array}$ & $\begin{array}{l}\text { Halocyntin and } \\
\text { papillosin }\end{array}$ & & Antimicrobial & [46] \\
\hline $\begin{array}{c}\text { Hemocytes } \\
\text { (Halocynthia aurantium) }\end{array}$ & Halocidin & & Antimicrobial & {$[47]$} \\
\hline Gonad (Unknown sp.) & GnRH-2 peptide & Pheromone-like function & Induce spawning & [48] \\
\hline
\end{tabular}

Microbes associated with tunicates have been found to produce potential metabolites showing antimicrobial and anticancer activities (Figures 1-3 and Table 3). Tunicateassociated bacteria such as Bacillus, Pantoea, Pseudoalteromonas, Salinicola, Streptomyces, Vibrio and Virgibacillus have recently been identified with potential antimicrobial activities [16]. The introduced tunicate species are also reported to harbor diverse host-specific microbial populations [49] that produce species-specific metabolites [50]. In general, tunicate associated bacteria and fungi are known to produce a variety of MNPs with various biological properties [41]. The chemistry of yellow pigment-producing parasitic bacteria in the interstitial and blood-filled spaces of planktonic tunicates, Oikopleura vanhoeffeni and Oikopleura dioica, are yet to be characterized [51].

Table 3. Bioactive compounds from various species of tunicates and their associated microbes.

\begin{tabular}{|c|c|c|c|c|}
\hline MNPs from Tunicates & Chemical Compound & Function & Application & Reference \\
\hline Aplidium albicans & Aplidin & & Anticancer & {$[52,53]$} \\
\hline Aplidium albicans & Dehydrodidemnin B & & Antitumor & [54] \\
\hline Aplidium glabrum & Quinones & & Anticancer, cytotoxic & {$[55]$} \\
\hline Aplidium haouarianum & Haouamine A & & Cytotoxic activity & [56] \\
\hline Aplidium meridianum & Meridianins & & Anticancer, antibiofilm & [57] \\
\hline Aplidium \& Synoicum & Meridianins & Feeding deterrents & Antibacterial & [58] \\
\hline Atapozoa sp. & Tambjamine & Feeding deterrents & & [59] \\
\hline Botryllus tuberatus & Tuberatolides & & $\begin{array}{l}\text { Farnesoid } X \text { receptor } \\
\text { antagonists }\end{array}$ & {$[60]$} \\
\hline Clavelina lepadiformis & Lepadins and villatamines & & $\begin{array}{l}\text { Antiparasitic, } \\
\text { anticancer }\end{array}$ & {$[61]$} \\
\hline Clavelina picta & Clavepictine A and B & & $\begin{array}{l}\text { Antimicrobial, } \\
\text { cytotoxicity }\end{array}$ & [62] \\
\hline Cynthia savignyi & Cynthichlorine & & Antifungal, cytotoxicity & [63] \\
\hline Cystodytes dellechiajei & Cystodytins A-I & & Antitumor, cytotoxic & {$[64,65]$} \\
\hline Cystodytes dellechiajei & Ascididemin & & Antitumor & {$[66]$} \\
\hline Cystodytes sp. & Ascididemin & Feeding deterrents & Antifeedant & [67] \\
\hline
\end{tabular}


Table 3. Cont.

\begin{tabular}{|c|c|c|c|c|}
\hline MNPs from Tunicates & Chemical Compound & Function & Application & Reference \\
\hline Didemnidae & $\begin{array}{l}\text { Mellpaladine and } \\
\text { dopargimine }\end{array}$ & & Neuroactive & [68] \\
\hline Didemnidae & Siladenoserinols A and B & & Antitumor & [69] \\
\hline Didemnidae & Sameuramide A & & Colony formation & {$[70]$} \\
\hline Didemnum sp. & Lepadins D-F & & $\begin{array}{l}\text { Antiplasmodial and } \\
\text { antitrypanosomal }\end{array}$ & [71] \\
\hline Didemnum guttatum & $\begin{array}{l}\text { Cyclodidemniserinol } \\
\text { trisulfate }\end{array}$ & & Anti-retroviral & [72] \\
\hline Didemnum granulatum & Granulatamides & & Deterrent activity & [73] \\
\hline Didemnum molle & Lanthipeptide divamide A & & anti-HIV drug & [74] \\
\hline Didemnum molle & Mollamide B & & Anticancer & {$[75]$} \\
\hline Didemnum proliferum & Shishijimicins & & Antitumor & {$[76]$} \\
\hline Didemnum psammatodes & Methyl esters & & Antiproliferative & [77] \\
\hline Didemnum ternerratum & Lamellarin Sulfates & & Anticancer & [78] \\
\hline Diplosoma sp. & Diplamine & & $\begin{array}{l}\text { Antibacterial and } \\
\text { cytotoxic }\end{array}$ & [79] \\
\hline Diplosoma virens & $\begin{array}{l}\text { Diplosoma ylidene } 1, \\
\text { Diplosoma ylidene } 2\end{array}$ & & Anticancer & [80] \\
\hline Ecteinascidia turbinata & $\begin{array}{l}\text { Ecteinascidin } 743 \\
\text { (Trabectedin) }\end{array}$ & & Anticancer & [81] \\
\hline Eudistoma gilboverde & Methyleudistomins & & Antitumor & [82] \\
\hline Eudistoma olivaceum & Eudistomins $\mathrm{G}$ and $\mathrm{H}$ & Chemical defense & Antifouling & [34] \\
\hline Eudistoma olivaceum & $\begin{array}{c}\text { Eudistomins } \mathrm{A}, \mathrm{D}, \mathrm{G}, \mathrm{H}, \mathrm{I}, \\
\mathrm{J}, \mathrm{M}, \mathrm{N}, \mathrm{O}, \mathrm{P} \text {, and } \mathrm{Q}\end{array}$ & & Antiviral & [83] \\
\hline Eudistoma olivaceum & Eudistomins $\mathrm{C}, \mathrm{E}, \mathrm{K}$, and L & & Antiviral & {$[84]$} \\
\hline Eudistoma vannamei & 7-Oxostaurosporine & & Anticancer & [85] \\
\hline Eudistoma viride & Eudistomins $\mathrm{H}$ & & Anticancer & {$[86]$} \\
\hline Eusynstyela latericius & Eusynstyelamides A, B & & Antibacterial & {$[87]$} \\
\hline Eusynstyela tincta & Kuanoniamine A & Chemical defense & $\begin{array}{c}\text { Antimicrobial, } \\
\text { antitumor, antifouling }\end{array}$ & [88] \\
\hline Halocynthia aurantium & Halocidin & & Antimicrobial & {$[47]$} \\
\hline Halocynthia papillosa & Halocyntin and papillosin & & Antimicrobial & {$[46]$} \\
\hline Halocynthia roretzi & Lumichrome & Larval metamorphosis & & [89] \\
\hline Halocynthia roretzi & Halocyamine A and B & & $\begin{array}{l}\text { Antimicrobial, } \\
\text { anticancer }\end{array}$ & [90] \\
\hline Lissoclinum cf. badium & Lissoclibadins & & Anticancer & [91] \\
\hline Lissoclinum fragile & & & $\begin{array}{c}\text { Antimicrobial, } \\
\text { hemolytic, and } \\
\text { cytotoxic }\end{array}$ & [92] \\
\hline Lissoclinum patella & Patellazole B and C & & $\begin{array}{c}\text { Antimicrobial, } \\
\text { cytotoxic }\end{array}$ & {$[93,94]$} \\
\hline Phallusia nigra & $\begin{array}{l}\text { Vanadium chloride, } \\
\text { vanadyl sulfate }\end{array}$ & & Antimicrobial & [95] \\
\hline
\end{tabular}


Table 3. Cont.

\begin{tabular}{|c|c|c|c|c|}
\hline MNPs from Tunicates & Chemical Compound & Function & Application & Reference \\
\hline Polycarpa aurata & Polyaurines A and B & & Antiparasitic & [96] \\
\hline Polycarpa clavata & $\begin{array}{c}\text { Polycarpine } \\
\text { dihydrochloride }\end{array}$ & & Cytotoxic & [97] \\
\hline Polycarpa clavata & Polycarpaurines $\mathrm{A}$ and $\mathrm{C}$ & & Antiviral, antifungal & [98] \\
\hline Polyclinidae & $\begin{array}{l}\text { Sodium 1-(12-hydroxy) } \\
\text { octadecanyl sulfate }\end{array}$ & & $\begin{array}{c}\text { Matrix } \\
\text { metalloproteinase } 2 \\
\text { inhibitor }\end{array}$ & [99] \\
\hline Polysyncraton lithostrotum & Namenamicin & & Cytotoxic, antitumor & [100] \\
\hline Polyandrocarpa sp. & Polyandrocarpidines & & $\begin{array}{c}\text { Antimicrobial, } \\
\text { cytotoxic, and deterrent } \\
\text { activities }\end{array}$ & {$[101,102]$} \\
\hline Polyandrocarpa misakiensis & Retinoic acid & & Regeneration of gut & [103] \\
\hline Pseudodistoma antinboja & Cadiolides J-M & & Antibacterial & [104] \\
\hline Pycnoclavella kottae & Kottamide D & & $\begin{array}{c}\text { Cytotoxic, } \\
\text { anti-inflammatory, and } \\
\text { antimetabolic activities }\end{array}$ & [105] \\
\hline Sidnyum turbinatum & Alkyl sulfates & & Antiproliferative & [106] \\
\hline Stolonica sp. & Stolonic acid A and B & & Antiproliferative & [107] \\
\hline Styela clava & Clavanins & & Antimicrobial & [108] \\
\hline Styela plicata & Hemocytes & & Cytotoxic & [109] \\
\hline Synoicum adareanum & $\begin{array}{l}\text { Hyousterones and } \\
\text { Abeohyousterone }\end{array}$ & & $\begin{array}{l}\text { Cytotoxic and } \\
\text { anticancer }\end{array}$ & [110] \\
\hline Trididemnum solidum & Didemnins A, B, and C & & Antiviral, cytotoxic & {$[111,112]$} \\
\hline \multicolumn{5}{|l|}{$\begin{array}{c}\text { MNPs from associated } \\
\text { microbes }\end{array}$} \\
\hline $\begin{array}{c}\text { Candidatus } \\
\text { Endoecteinascidia } \\
\text { frumentensis }\end{array}$ & Tetrahydroisoquinoline & & & [113] \\
\hline Microbulbifer sp. & Bulbiferates A and B & & Antibacterial & [114] \\
\hline Penicillium verruculosum & $\begin{array}{c}\text { Verruculides A, } \\
\text { chrodrimanins A and } \mathrm{H}\end{array}$ & & $\begin{array}{c}\text { Protein tyrosine } \\
\text { phosphatase 1B } \\
\text { inhibition }\end{array}$ & [115] \\
\hline Pseudoalteromonas rubra & Isatin & Microbial defense & Antibacterial & [16] \\
\hline Pseudoalteromonas tunicata & Tambjamine & Feeding deterrents & & [116] \\
\hline Pseudoalteromonas tunicata & Tambjamine & & Antifungal & [117] \\
\hline Pseudovibrio denitrificans & Diindol-3-ylmethanes & & Antifouling & [118] \\
\hline Saccharopolyspora sp. & JBIR-66 & & Cytotoxic & [119] \\
\hline Serratia marcescens & Tetrapyrrole pigment & Feeding deterrents & & [120] \\
\hline Streptomyces sp. & $\begin{array}{l}\text { Granaticin, granatomycin } \\
\text { D, and dihydrogranaticin B }\end{array}$ & & Antibacterial & [121] \\
\hline Talaromyces sp. & Talaropeptides A-D & & $\begin{array}{c}\text { Plasma stability, } \\
\text { Antibacterial, } \\
\text { antifungal, cytotoxic }\end{array}$ & [24] \\
\hline $\begin{array}{l}\text { Tistrella mobilis and } \\
\text { Tistrella bauzanensis }\end{array}$ & Didemnin & & Anticancer & {$[23,122]$} \\
\hline
\end{tabular}




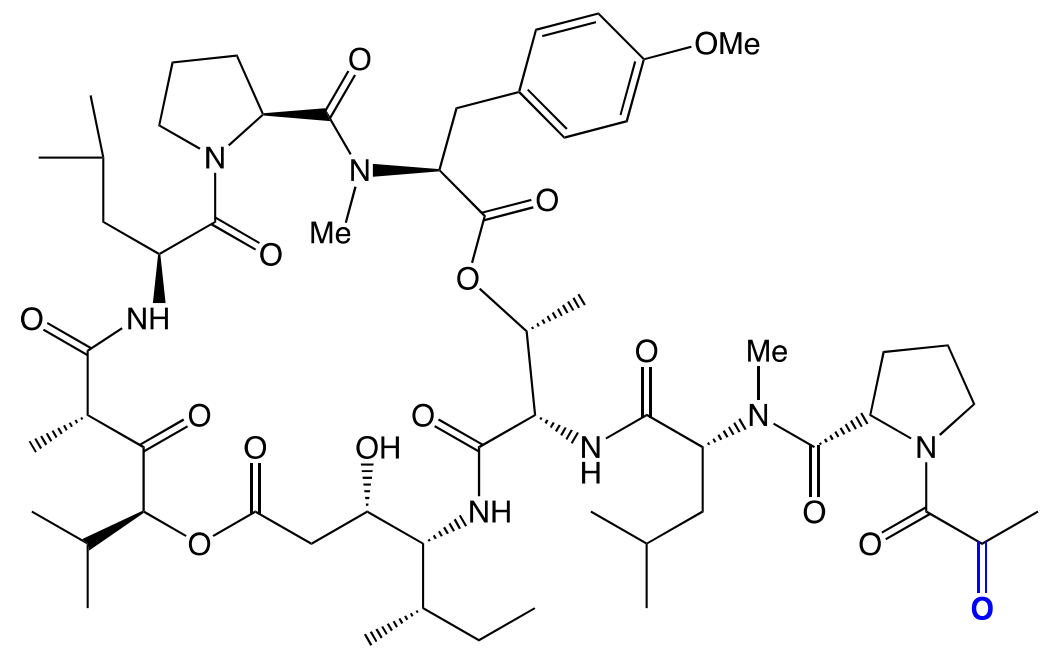

Plitidepsin

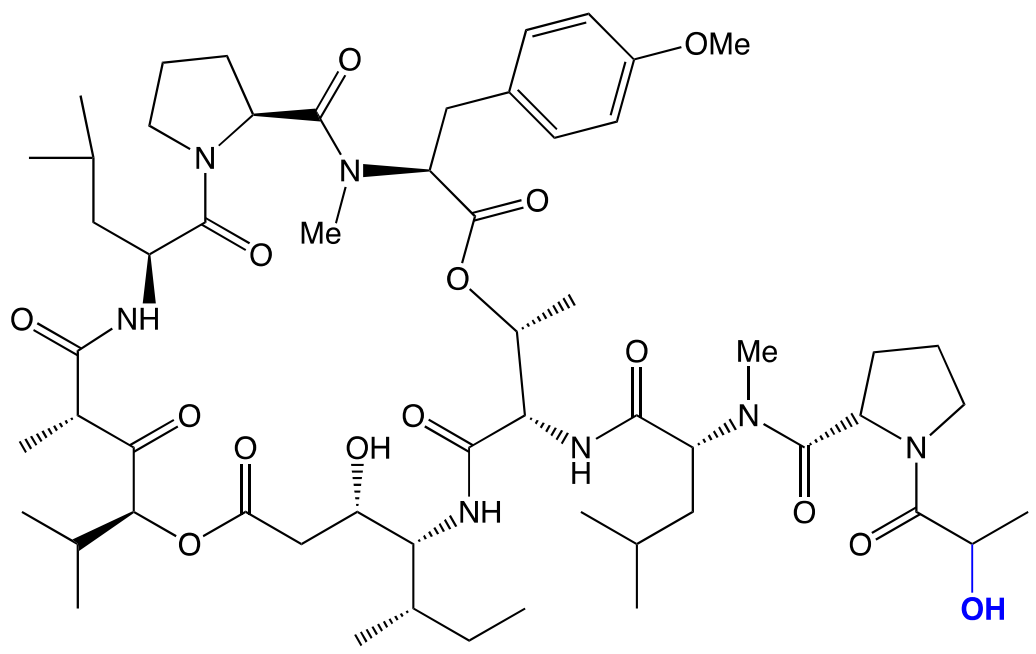

Didemnin B



Figure 1. Important anticancer drugs of tunicates and their associated microbes in clinical trials. 


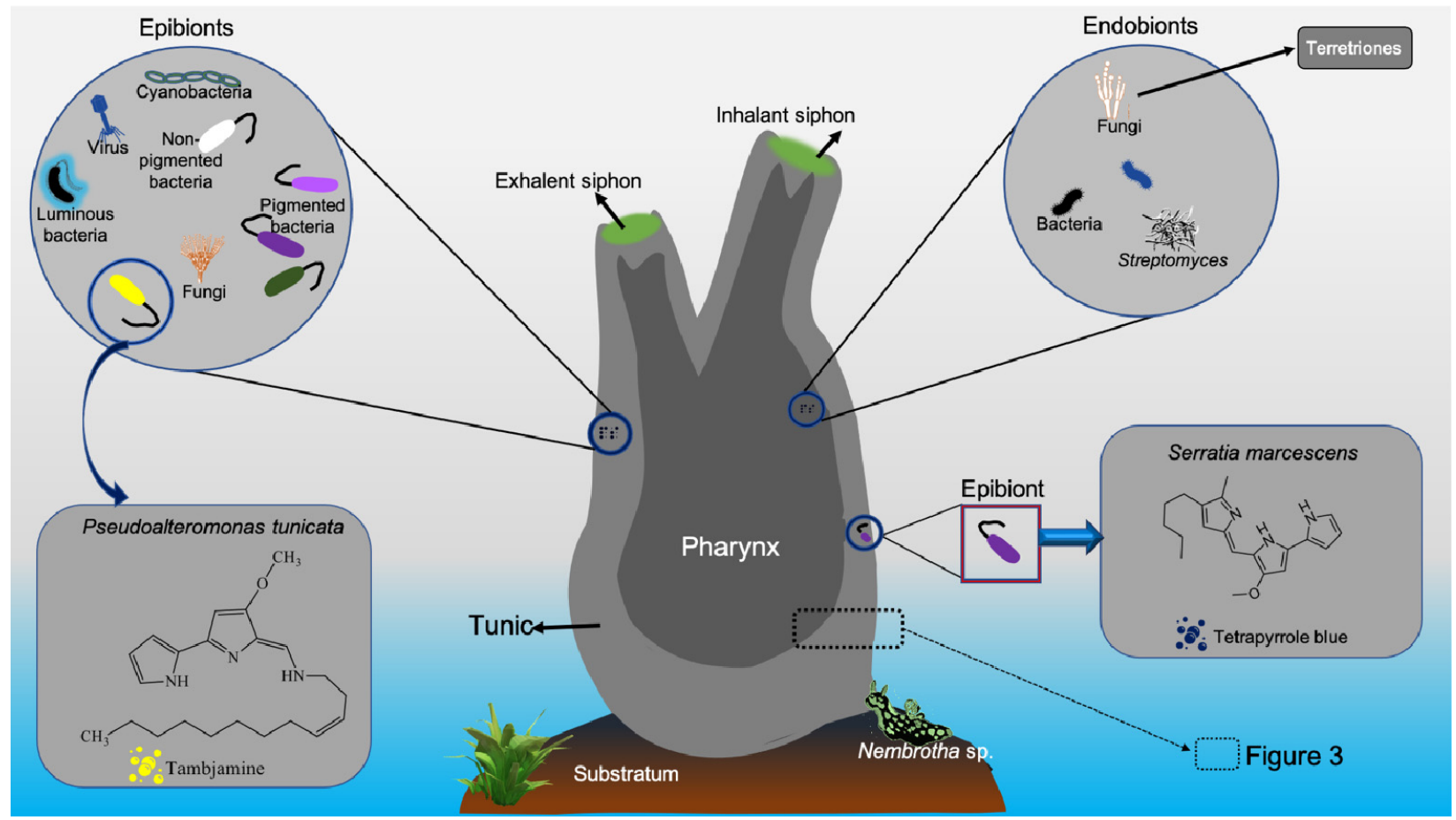

Figure 2. Tunicate-associated epibiotic and endobiotic symbionts. (the small inserted empty box provides more details in Figure 3).

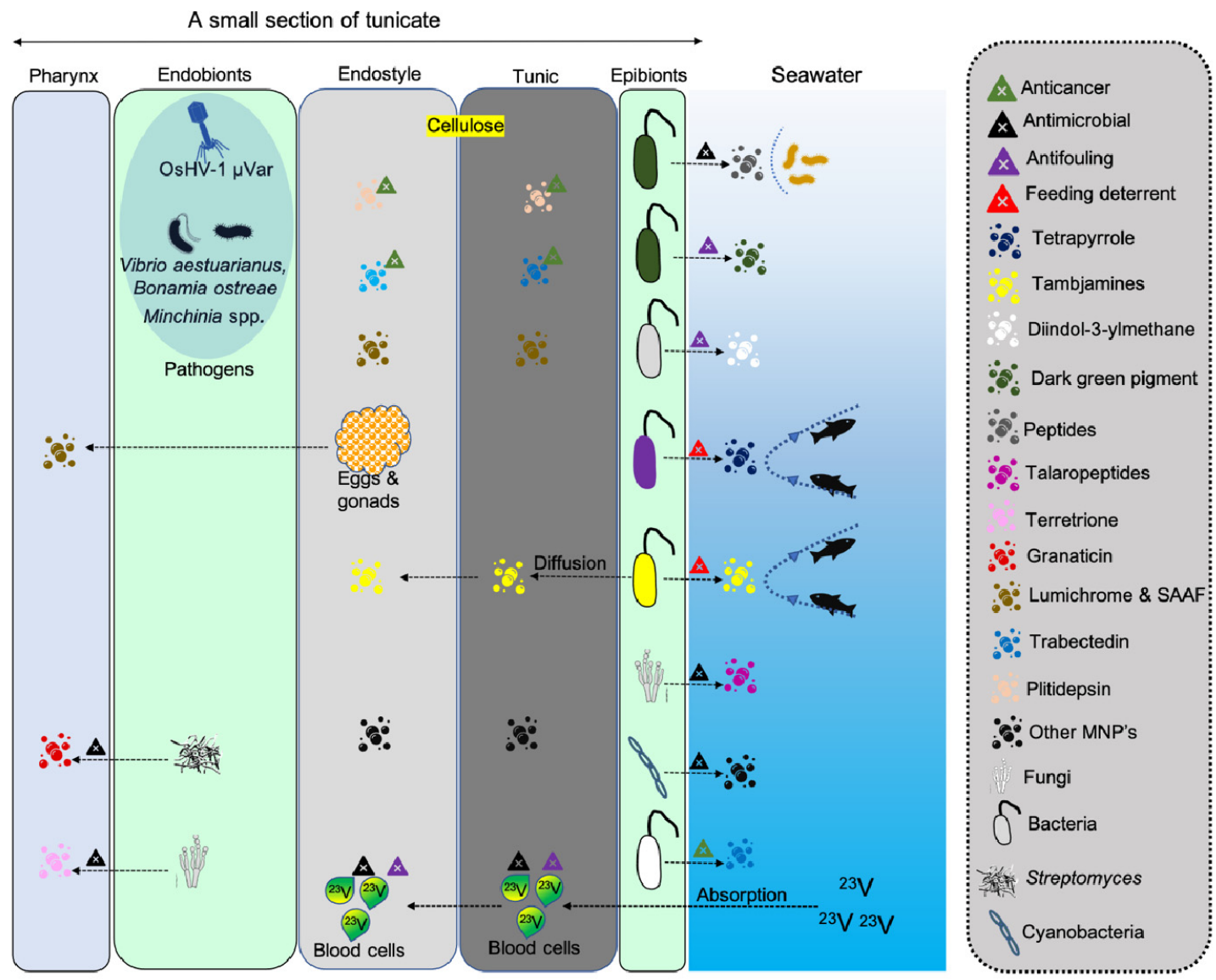

Figure 3. Illustration depicting various MNPs released from endobiotic and epibiotic microbes associated with tunicate's endostyle and tunic. 


\section{Antimicrobial Applications}

Tunicates [123], with their associated epi-symbionts [16,124] and endosymbionts [125], are prolific producers of antimicrobial and antifungal compounds inhibiting pathogens. The brominated alkaloids [126] and other compounds from tunicates have been reported to possess several biological activities $[25,26]$. Pseudoalteromonas tunicata produces alkaloid tambjamine (425 nm), an antifungal yellow pigment $[127,128]$, and violacein $(575 \mathrm{~nm})$, a purple pigment with antiprotozoal activity $[129,130]$, in addition to a range of bioactive compounds $[129,131]$. Methanol extraction of Lissoclinum fragile displayed antibacterial, antifungal, hemolytic, and cytotoxic activities [92]. The kuanoniamine A metabolite produced by Eusynstyela tincta inhibited pathogenic bacteria such as B. subtilis, E. coli, S. aureus, $V$. cholerae, and V. parahaemolyticus and fungi A. fumigatus and C. albicans [88]. A diffusible 190-kDa protein produced by tunicate Ciona intestinalis associated bacterium Pseudoalteromonas tunicata was found to show antibacterial activity against marine isolates [132]. The four $\alpha$-helical peptides "clavanins A, B, C, and D" isolated from the hemocytes of tunicate Styela clava showed antibacterial activity against pathogenic Listeria monocytogenes strain EGD and antifungal activity against Candida albicans [44]. Halocidin, an antimicrobial peptide purified from tunicate Halocynthia aurantium showed antibacterial activity against methicillin-resistant Staphylococcus aureus and multidrug-resistant $P_{s e u-}$ domonas aeruginosa [47]. Similarly, halocyntin and papillosin peptides isolated from tunicate Halocynthia papillosa also displayed antibacterial activity against both Gram-positive and Gram-negative marine bacteria [46]. Halocyamine peptides synthesized by the hemocytes of Halocynthia roretzi showed antimicrobial activity against various bacteria and yeasts [90]. Similarly, Halocyamines produced by Styela clava also displayed antimicrobial properties [108]. A salt-tolerant peptide isolated from hemocytes of Ciona intestinalis showed both antibacterial and antifungal activity [133]. Vanadium chloride and vanadyl sulfate also displayed antibacterial activity against various pathogens [95].

An endobiont, Streptomyces sp., isolated from the tunicate, Styela canopus, produced antibacterial compounds such as granaticin, granatomycin D, and dihydrogranaticin B [121]. Similarly endosymbiotic fungi associated with the tunicates, Polycarpa aurata [134] and Rhopalaea crassa [135], showed antimicrobial activity. The fungi Talaromyces sp., isolated from an unidentified tunicate, produced talaropeptides A and B, two antibacterial metabolites that inhibited Gram-positive bacteria, Bacillus subtilis [24]. The endophytic fungus Penicillium sp. isolated from Didemnum sp. produced antifungal and cytotoxic compounds, terretrione C and D [136].

Some tunicates produced antiviral molecules, indicating their chemical defense function against environmental viruses. The Caribbean tunicate, Trididemnum sp., was found to produce depsipeptides, particularly didemnin A and B, exhibiting antiviral activity against DNA and RNA viruses in vitro $[111,137]$. Another species of Caribbean tunicate, Eudistoma olivaceum, produced prolific MNPs, such as eudistomins A, D, G, H, I, J, M, N, O, P, and $\mathrm{Q}$, which possessed antiviral activity [83]. The ascidian Didemnum guttatum was found to produce the cyclodidemniserinol trisulfate compound that showed anti-retroviral activity by inhibiting HIV-1 integrase [72]. The tunicate, Didemnum molle, released lanthipeptide divamide A that promised to be a potential anti-HIV drug [74] (Table 4).

\section{Anticancer and Antitumor Applications}

Trabectedin (Ecteinascidin; ET-743; Yondelis ${ }^{\circledR}$ ), an alkaloid extracted from the orange tunicate, Ecteinascidia turbinata, is approved as a first anticancer drug [138] to treat breast cancer [139,140], soft tissue sarcoma [141], and ovarian cancer [142-144]. This molecule is suggested to originate from E. turbinata symbiotic bacteria, Candidatus Endoecteinascidia frumentensis [145]. However, plitidepsin $\left(\right.$ Aplidin $^{\circledR}$ ), a depsipeptide isolated from the Mediterranean tunicate, Aplidium albicans, is in phase II clinical trials $[138,146]$ as an anticancer drug against breast cancer [147], human kidney carcinoma cells [52], and multiple myeloma [53]. Didemnin B is also in phase II trials [148], showing anticancer activity against leukaemia P388 cells [111]. Significantly, 60\% of the human cervical carcinoma cell lines (HeLa) 
were inhibited by Eudistomins $\mathrm{H}$ extracts $\left(\mathrm{IC}_{50} 0.49 \mu \mathrm{g} / \mathrm{mL}\right.$ ) obtained from E. viride [86]. Clavepictine A and B alkaloids originated from Clavelina picta demonstrated potential cytotoxic activity ( $\mathrm{IC}_{50} 12 \mu \mathrm{g} / \mathrm{mL}$ ) against murine leukemia and human solid tumor cell lines [62]. Lamellarin sulfates originated from Didemnum ternerratum [78] and polycarpine dihydrochloride, a disulfide alkaloid extracted from an ascidian Polycarpa clavata, were found to inhibit human colon tumor cell lines [97].

Cystodytins A, B, and C, three teracyclic alkaloids isolated from Okinawa tunicate Cystodytes dellechiajei, were reported to show antitumor activities [64]. Macrolides isolated from tunicates Lissoclinum patella (Patellazole C) [94] and Eudistoma cf. rigida (Lejimalides A, B, C, and D) [149,150] possessed anticancer activity [151]. Diplamine, an orange pigment alkaloid produced by Diplosoma sp., demonstrated cytotoxic activity against leukemia cells [79]. Halocyamine A and B peptides extracted from $H$. roretzi showed anticancer activity against various cell lines [90]. A depsipeptide, dehydrodidemnin B, produced by Aplidium albicans inhibited Ehrlich carcinoma cells in mice and reduced $80-90 \%$ tumor cells [54]. Bryostatins Ecteinascidins products, such as ET-729, 743, 745, 759 A, 759B, and 770, extracted from the Caribbean tunicate Ecteinascidia turbinata showed immunomodulator activity and antitumor activity against various leukemia cells [152] and breast, lung, ovary, and melanoma cells [153]. The Brazilian ascidian, Didemnum granulatum, produced G2 checkpoint-inhibiting aromatic alkaloids, granulatimide and isogranulatimide [154]. The ascidian Cystodytes dellechiajei produced topoisomerase II-inhibiting ascididemin, which has antitumor activity against various tumor cell lines [66]. This marine alkaloid exhibits marked cytotoxic activities against a range of tumor cells. The kuanoniamine A metabolite extracted from E. tincta displayed 100\% inhibition of Dalton's lymphoma and Ehrlich ascites tumor cell lines [88]. Cynthichlorine, an alkaloid isolated from the tunicate Cynthia savignyi, showed cytotoxicity against Artemia salina larva at an $\mathrm{LD}_{50}$ of $48.5 \mu \mathrm{g} / \mathrm{mL}$ [63]. Siladenoserinols A and B derivatives isolated from didemnid tunicates possessed antitumor activity by inhibiting the interaction of p53-Hdm2 [69] (Table 4).

Table 4. Bioactive MNP's from tunicates and associated microbes.

\begin{tabular}{|c|c|c|c|c|c|c|}
\hline Application & Compound & Activity against & $\begin{array}{c}\text { Dose/ } \\
\text { Concentration }\end{array}$ & $\begin{array}{c}\text { Growth } \\
\text { Inhibition } \\
\text { (Diameter/ } \\
\text { Percentage) }\end{array}$ & $\begin{array}{l}\text { Assay } \\
\text { Method }\end{array}$ & Reference \\
\hline \multicolumn{7}{|l|}{ Antimicrobial } \\
\hline & Clavanins & $\begin{array}{l}\text { E. coli, } \\
\text { L. monocytogenes, } \\
\text { C. albicans }\end{array}$ & $1.6-3.5 \mu \mathrm{g} / \mathrm{mL}$ & - & $\begin{array}{l}\text { Radial } \\
\text { diffusion } \\
\text { assay }\end{array}$ & [44] \\
\hline & Diplamine & $\begin{array}{c}\text { E. coli, } \\
\text { S. aureus }\end{array}$ & & & - & [79] \\
\hline & Halocidin & $\begin{array}{l}\text { Methicillin-resistant } \\
\text { Staphylococcus aureus and } \\
\text { multidrug-resistant } \\
\text { Pseudomonas aeruginosa }\end{array}$ & $100-200 \mu \mathrm{g} / \mathrm{mL}$ & 5-11 mm & $\begin{array}{l}\text { Radial } \\
\text { diffusion } \\
\text { assay }\end{array}$ & [47] \\
\hline & Isatin & $\begin{array}{c}\text { Bacillus cereus, Bacillus } \\
\text { megaterium, Escherichia coli, } \\
\text { Micrococcus luteus, }\end{array}$ & MIC $200 \mu \mathrm{g} / \mathrm{mL}$ & $7->21 \mathrm{~mm}$ & $\begin{array}{l}\text { Disk } \\
\text { diffusion } \\
\text { assay }\end{array}$ & [16] \\
\hline & $\begin{array}{c}\text { Kuanoniamine } \\
\text { A }\end{array}$ & $\begin{array}{c}\text { B. Subtilis, E. coli, S. aureus, } \\
\text { V. cholerae, } \\
\text { V. parahaemolyticus and } \\
\text { fungus } \\
\text { A. jumigatus and C. albicans }\end{array}$ & $25 \mu \mathrm{g} / \mathrm{mL}$ & $7-13 \mathrm{~mm}$ & $\begin{array}{l}\text { Disk } \\
\text { diffusion } \\
\text { assay }\end{array}$ & [88] \\
\hline & Cynthichlorine & $\begin{array}{c}\text { A. radiobacter, } \\
\text { E. coli, } \\
\text { P. aeruginosa, } \\
\text { Botrytis cinerea, } \\
\text { Verticillium albo atrum }\end{array}$ & & 6-10 mm & $\begin{array}{l}\text { Disc } \\
\text { diffusion } \\
\text { assay }\end{array}$ & [63] \\
\hline
\end{tabular}


Table 4. Cont.

\begin{tabular}{|c|c|c|c|c|c|c|}
\hline Application & Compound & Activity against & $\begin{array}{c}\text { Dose/ } \\
\text { Concentration }\end{array}$ & $\begin{array}{l}\text { Growth } \\
\text { Inhibition } \\
\text { (Diameter/ } \\
\text { Percentage) }\end{array}$ & $\begin{array}{l}\text { Assay } \\
\text { Method }\end{array}$ & Reference \\
\hline & $\begin{array}{c}\text { Talaropeptides A } \\
\text { and B }\end{array}$ & Bacillus subtilis & $\mathrm{IC}_{50} 1.5-3.7 \mu \mathrm{M}$ & $50 \%$ & $\begin{array}{l}\text { Microtiter } \\
\text { plate assay }\end{array}$ & [24] \\
\hline & $\begin{array}{l}\text { Terretrione } \mathrm{C} \text { and } \\
\mathrm{D}\end{array}$ & Candida albicans & MIC $32 \mu \mathrm{g} / \mathrm{mL}$ & $17-19 \mathrm{~mm}$ & $\begin{array}{l}\text { Disc } \\
\text { diffusion } \\
\text { assay }\end{array}$ & [136] \\
\hline \multicolumn{7}{|l|}{$\begin{array}{c}\text { Anticancer \& } \\
\text { antitumor }\end{array}$} \\
\hline & Aplidin & $\begin{array}{l}\text { Multiple myeloma cell lines, } \\
\text { MDA-MB-231 breast cancer } \\
\text { cells, A-498 and ACHN } \\
\text { cell lines }\end{array}$ & $\mathrm{IC}_{50} 1$ to $15 \mathrm{nmol} / \mathrm{L}$ & & $\begin{array}{c}\text { Nuclear } \\
\text { Staining } \\
\text { Assay; MTT } \\
\text { assay }\end{array}$ & {$[52,53]$} \\
\hline & $\begin{array}{l}\text { Clavepictines A } \\
\text { and B }\end{array}$ & $\begin{array}{l}\text { Murine leukemia and } \\
\text { human solid tumor cell lines }\end{array}$ & $\mathrm{IC}_{50} 12 \mu \mathrm{g} / \mathrm{mL}$ & & $\begin{array}{l}\text { Microculture } \\
\text { tetrazolium } \\
\text { assay }\end{array}$ & [62] \\
\hline & $\begin{array}{l}\text { Dehydrodidemnin } \\
\text { B }\end{array}$ & Ehrlich carcinoma cells & $2.5 \mu \mathrm{g} /$ mouse & $70-90 \%$ & MTT assay & [54] \\
\hline & $\begin{array}{l}\text { Didemnins A and } \\
\text { B }\end{array}$ & Leukaemia P388 cells & $\mathrm{IC}_{50} 1.5-25 \mu \mathrm{g} / \mathrm{mL}$ & & - & [111] \\
\hline & Diplamine & Leukemia L1210 cells & $\begin{array}{c}\mathrm{IC}_{50} \\
2 \times 10^{-2} \mu \mathrm{g} / \mathrm{mL}\end{array}$ & & - & [79] \\
\hline & $\begin{array}{l}\text { Ecteinascidin } 743 \\
\text { (Trabectedin) }\end{array}$ & Leukemia L1210 cells & $\mathrm{IC}_{50} 0.5 \mu \mathrm{g} / \mathrm{mL}$ & & - & [152] \\
\hline & Eudistomins $\mathrm{H}$ & HeLa cell lines & $\mathrm{IC}_{50} 0.49 \mu \mathrm{g} / \mathrm{mL}$ & $60 \%$ & MTT assay & [86] \\
\hline & $\begin{array}{l}\text { Halocyamine } \mathrm{A} \\
\text { and } \mathrm{B}\end{array}$ & $\begin{array}{l}\text { Rat neuronal cells, mouse } \\
\text { neuroblastoma N-18 cells, } \\
\text { and human Hep-G2 cells }\end{array}$ & & & - & [90] \\
\hline & Kuanoniamine A & $\begin{array}{c}\text { Dalton's lymphoma and } \\
\text { Ehrlich ascites tumour cell } \\
\text { lines }\end{array}$ & $25 \mu \mathrm{g} / \mathrm{mL}$ & $100 \%$ & $\begin{array}{l}\text { Trypan blue } \\
\text { exclusion test }\end{array}$ & [88] \\
\hline & $\begin{array}{l}\text { Lamellarin } \\
\text { Sulfates }\end{array}$ & $\begin{array}{l}\text { HCT-116 human colon } \\
\text { tumor cells }\end{array}$ & $\mathrm{IC}_{50} 9.7 \mu \mathrm{M}$ & & $\begin{array}{l}\text { MTS cell } \\
\text { proliferation } \\
\text { assay }\end{array}$ & [78] \\
\hline & Namenamicin & $\begin{array}{c}\text { P388 leukemia cells, 3Y1, } \\
\text { and HeLa }\end{array}$ & $\begin{array}{c}\mathrm{IC}_{50} 3.5 \mathrm{nM} ; \\
\mathrm{IC}_{50} 3.3-13 \mathrm{pM}\end{array}$ & & $\begin{array}{l}\text { Biochemical } \\
\text { prophage } \\
\text { induction } \\
\text { assay }\end{array}$ & [100] \\
\hline & $\begin{array}{c}\text { Polycarpine } \\
\text { dihydrochloride }\end{array}$ & $\begin{array}{l}\text { HCT-116 human colon } \\
\text { tumor cells }\end{array}$ & $\mathrm{ED}_{50} 1.9 \mu \mathrm{g} / \mathrm{mL}$ & & - & [97] \\
\hline & $\begin{array}{c}\text { 7- } \\
\text { oxostaurosporine }\end{array}$ & $\begin{array}{l}\text { HL-60, Molt-4, Jurkat, K562, } \\
\text { HCT-8, MDA MB-435, and } \\
\text { SF-295 cell lines }\end{array}$ & $\mathrm{IC}_{50} 10-58 \mathrm{nM}$ & $95 \%$ & MTT assay & [85] \\
\hline & $\begin{array}{l}\text { Terretrione } C \text { and } \\
\text { D }\end{array}$ & Human breast cancer cells & $\begin{array}{c}\mathrm{IC}_{50} 16.5 \text { and } \\
17.6 \mu \mathrm{M}\end{array}$ & & $\begin{array}{l}\text { Sulforhodamine } \\
\text { B assay }\end{array}$ & [136] \\
\hline \multicolumn{7}{|l|}{ Antifouling } \\
\hline & $\begin{array}{l}\text { Diindol-3- } \\
\text { ylmethanes }\end{array}$ & $\begin{array}{c}\text { Barnacle, Balanus amphitrite } \\
\text { and bryozoan, } \\
\text { Bugula neritina }\end{array}$ & $\mathrm{EC}_{50} 18.57$ & & $\begin{array}{l}\text { Microtiter } \\
\text { plate assay }\end{array}$ & [118] \\
\hline & $\begin{array}{l}\text { Eudistomins } G \\
\text { and } H\end{array}$ & Fish and other larvae & & & $\begin{array}{l}\text { Antifeedant } \\
\text { assay }\end{array}$ & [34] \\
\hline
\end{tabular}




\section{Antifouling and Anti-Deterrent Activities}

The colonial tunicate, Eudistoma olivaceum, was found to produce brominated alkaloids, Eudistomins $\mathrm{G}$ and $\mathrm{H}$, which acted as antifouling substances and fish antifeedants; thus, the E. olivaceum surface was completely free from fouling epibionts [34]. A dark green pigmented bacteria, Pseudoalteromonas tunicata, isolated from the surface of Ciona intestinalis, collected originally from off the west coast of Sweden, showed antifouling activity against algal spores, invertebrate larvae, and diatoms [131,155,156]. The yellow pigmented Pseudoalteromonas tunicata mutants have demonstrated antifouling activity against algal spore germination, bacterial growth, fungal growth, and invertebrate larvae [129]. Diindol3-ylmethane products isolated from an unidentified ascidian-associated bacteria, Pseudovibrio denitrificans, displayed nearly $50 \%$ antifouling activity against barnacle Balanus amphitrite and bryozoan Bugula neritina [118].

Deterring activity of vanadium acidic solutions, such as vanadyl sulfate and sodium vanadate, was observed against Thalassoma bifasciatum when incorporated into food pellets [95,157]. Didemnimides C and D from Didemnum conchyliatum [158], nordidemnin B [102] and didemnin B [159] from Trididemnum solidum, and granulatamides from Didemnum granulatum [73] displayed antifeedant effects on various fishes in laboratory experiments. The kuanoniamine A molecule from E. tincta displayed feeding-deterrent activities against carnivore gold fish, Carassius auratus [88]. MNPs isolated from Antarctic tunicates have demonstrated variability in anti-deterrent activities [58]. Both the yellow pigmented tambjamine metabolites and blue tetrapyrrole metabolite released from Sigillina sp. (i.e., Atapozoa sp.) showed feeding-deterrent activity against various carnivore fishes [59,160]. The blue tetrapyrrole pigment was suggested to originate from the associated bacteria Serratia marcescens [120]. Tambjamines and tetrapyrrole chemical constituents from both adult and larvae were reported to function as defensive chemicals against predators [102]. Lipophilic crude extracts from Antarctic tunicate, Distaplia cylindrica [161], and polyandrocarpidines from Polyandrocarpa sp. [101,102] demonstrated deterrent activity against certain sea-stars, hermit crabs, and snails (Table 4).

\section{Miscellaneous Applications}

The chiton Mopalia sp. spawned when injected with $1.0 \mathrm{mg} / \mathrm{L}$ of gonadotropin releasing hormone (GnRH2) of a tunicate [48]. Lumichrome, a compound extracted from tunic, gonads, and eggs of ascidian, Halocynthia roretzi, was involved in the larval metamorphosis [89]. Similarly, sperm-activating and attracting factors (SAAF) were isolated from eggs of the ascidians Ciona intestinalis and Ascidia sydneiensis [162]. Lipids extracted from $H$. roretzi have demonstrated the antidiabetic and anti-obese properties in mice models [163]. Two novel alkaloids, mellpaladine and dopargimine, isolated from Palauan tunicate have demonstrated neuroactive behavior in mice [68]. Two new alkaloids, polyaurines A and B, isolated from the tunicate, Polycarpa aurata, inhibited blood-dwelling Schistosoma mansoni [96]. Lepadin and villatamine alakaloids isolated from Clavelina lepadiformis [61] and lepadins from Didemnum sp. [71] displayed potential antiparasitic and cytotoxic activities. The ascidian species, Didemnum psammathodes, collected from the central west coast of India was extracted in organic solvents. These extracts showed antimicrobial and antifouling properties [164].

\section{Issues in Extraction \& Identification of Tunicate MNPs}

Marine organisms have developed diverse secondary metabolic pathways, which produce a vast number of unusual chemical moieties. These compounds belong to a wide variety of chemical classes, including terpenes, shikimates, polyketides, peptides, alkaloids, and many unidentified and uncharacterized structures (Houssen and Jaspars, 2012). There are several technologies in place to isolate and characterize the natural products from even a very small quantity of marine organisms. However, there are still hurdles in the isolation and characterization of bioactive molecules from ascidians. These include 1. taxonomic uncertainty: worldwide, there are very few taxonomists available for proper taxonomic 
assignments of tunicates. Sometimes the identification using molecular tools has been complicated by the difficulty in getting pure gDNA from the target species due to complex biotic associations (Houssen amd Jaspars, 2012). 2. Quantity of isolated molecules: most of the time, a small quantity of metabolites is available in the organisms, which is not even sufficient for spectroscopic analysis. 3. Instability of molecules: there are extremely labile compounds in the extracts, which decompose during the purification process, and we get artefacts. Of course, these problems are common in other marine invertebrates as well. Research funding has also become a hurdle for many young researchers; thus, many researchers are publishing their works with crude extracts instead of analyzing complete structural elucidation. If we could address these issues, we will be able to isolate and characterize novel bioactive molecules from this unique group of marine invertebrates. The quantity of molecules can be increased if we collect the target tunicate species at the right time (season) from the correct geographic location. This can be achieved by understanding the chemical ecology of the producing species. For this purpose, there should be joint efforts from marine biologists, ecologists, and natural product chemists.

\section{Metabolic Origin of Some Tunicates and Their Predators}

Several bioactive MNPs extracted from tunicates were believed to be originated from tunicates themselves. However, few studies have investigated the original origin of tunicate MNPs from their symbiotic microbes. Tambjamine pigments have been reported to be originated from tunicate-associated symbiotic bacteria like S. marcescens [160] and Pseudoalteromonas tunicata $[116,131]$. An identical dark blue pigmented tetrapyrrole compound isolated from an ascidian was observed from a bacterium [165]. The blue tetrapyrrole pigment was reported to have originated from the associated bacteria, Serratia marcescens [120]. Didemnins extracted from the tunicate, T. solidum [111], are found to be released by associated bacteria, Tistrella mobilis and Tistrella bauzanensis [23,122]. Similarly, the trabectedin compound identified from the Caribbean tunicate, E. turbinata [152,166], has now been observed to be produced by its symbiotic bacteria, Candidatus Endoecteinascidia frumentensis [145]. Meridianins isolated from Antarctic tunicates, Aplidium, Synoicum, and some sponges, are thought to have originated from their symbiotic microbes [58]. Similarly, tetrahydroisoquinoline constituents identified from the tunicate, Ecteinascidia turbinata, appeared to be released by the unculturable endosymbiotic bacterium, Candidatus Endoecteinascidia frumentensis [113]. Some of the bioactive MNPs identified from Didemnid tunicates also originated from their symbiotic cyanobacterial species, such as Synechocystis and Prochloron $[167,168]$. Namenamicin produced by the orange color ascidian, Polysyncraton lithostrotum, was suggested to originate from its symbiotic bacterium, Micromonospora species [100]. The anti-HIV lanthipeptide, divamide A, isolated from the tunicate, Didemnum molle, was found to be produced by uncultivable symbiotic bacteria [74].

Tunicates are known to produce more than 300 alkaloid compounds [126]. The tunicate predatory flatworm Prostheceraeus villatus was reported to obtain alkaloids, lepadins, and villatamines by preying (dietary origin) on the tunicate, Clavelina lepadiformis [61]. Likewise, tambjamine alkaloids observed in the ascidian Atapozoa sp. [160] and associated bacteria [131] were found to be acquired by the predatory nudibranchs, like Nembrotha sp., for defense functions [59,169]. Pyridoacridine metabolites observed in ascidians and some sponges indicate a possible microbial origin or convergent evolution of these molecules [170].

\section{Utilization of Invasive Tunicates Resources}

Tunicates usually occur in relatively low abundance in coastal waters. However, some tunicates are reported as invasive species in some coastal waters [171] and are known to cause space competition [172], damage to aquaculture $[173,174]$ by harboring pathogenic viruses and bacteria [175], and ecosystem alteration within the spread area [176]. Few non-invasive tunicate species of the coral reef environment have also been reported to overgrow on massive corals and caused minimal [112] or partial inhibition or delayed 
development of coral polyps [177]. A study reported the outbreak of the invasive tunicate, Diplosoma similis, that overgrew on corals and macrophytes and resulted in 50\% mortality of corals [178] (Table 5).

Table 5. Occurrence of invasive tunicate species in the global ocean and their impact on the marine ecosystem.

\begin{tabular}{|c|c|c|c|c|}
\hline Invasive Tunicate & Country & Origin Type & Negative Impacts & Reference \\
\hline Ascidiella aspersa & Argentina & Exotic & Space competition & [179] \\
\hline Botrylloides violaceus & Netherlands & Exotic & Space competition & [172] \\
\hline Botryllus schlosseri & Netherlands & Indigenous & Space competition & [172] \\
\hline $\begin{array}{l}\text { Botryllus schlosseri, } \\
\text { Botrylloides violaceus, } \\
\text { Ciona intestinalis, } \\
\text { Ciona savignyi, } \\
\text { Didemnum vexillum, } \\
\text { Molgula manhattensis, } \\
\text { Styela clava }\end{array}$ & USA & Exotic & $\begin{array}{l}\text { Competitors for food } \\
\text { and space }\end{array}$ & {$[180,181]$} \\
\hline Ciona intestinalis & Canada & Exotic & Mussel mortality & [176] \\
\hline Ciona intestinalis & Korea & Exotic & $\begin{array}{l}\text { Space competition and } \\
\text { damage to aquaculture }\end{array}$ & [174] \\
\hline Didemnum psammathodes & India & Indigenous & Space competition & [182] \\
\hline Didemnum vexillum & USA & Exotic & Threat to eelgrass & [183] \\
\hline Didemnum vexillum & Wales & Exotic & Space competition & [184] \\
\hline Diplosoma similis & American Sāmoa & Indigenous & Kill corals & [178] \\
\hline
\end{tabular}

Therefore, such overwhelming invasive species may be utilized to investigate their biological properties, biotechnological implications, and drug development. The exploitation of antiviral and cytotoxic didemnins from the invasive tunicate, T. solidum, has already been investigated [111,112]. Antimicrobial activity of $\alpha$-helical peptides "Clavanins" was identified from the hemocytes of the tunicate, Styela clava [44]. Thus, other invasive species need to be investigated for their bioactive properties. Seasonal studies on the spread of various invasive tunicates and their biomass estimations are an important research aspect for resource management and coastal conservation. A study suggested that ocean warming is triggering the rise of invasive species in coastal waters [185]. Therefore, identifying the key ocean-warming factors and their mitigation strategies is essential for a sustainable management of the global ocean bioresources.

\section{Research Gaps and Future Perspective}

Tunicates have been an important marine drug reservoir to treat a variety of diseases, including cancer. These resources from the ocean, particularly from the deep-sea, remain untapped for drug discovery. Therefore, exploration and exploitation of tunicate resources from coastal waters to the deep-sea and tropical to polar regions would open new insights in the drug discovery and evolutionary lineages. However, these efforts should be driven by chemical ecology of these organisms. The study of chemical ecology will help in bioprospecting and the efficient production of marine drugs from this unique group of organisms. On the other hand, the mode of colonization and pigment biosynthesis by associated microbes and the acquisition mechanism of pigments (e.g., tambjamines) by tunicates from their associated microbes are yet to be unveiled. Since tunicates have been reported to be colonized by pathogenic bacteria during filter feeding, the pathological implications of tunicates needs to be investigated to understand the possible transfer ways of pathogenic bacteria from tunicates to other biota and aquaculture setups. Therefore, regular biodiversity monitoring and population dynamics of tunicate resources should be performed to understand their distribution patterns and impact on the coastal resources. 
Author Contributions: Conceptualization, C.R., L.D., B.R.T. and M.R.; writing-original draft preparation, C.R., B.R.T., M.R., N.T. and L.D.; writing-review and editing, C.R., B.R.T., M.R., N.T. and L.D.; visualization, C.R. All authors have read and agreed to the published version of the manuscript.

Funding: This research was funded by CSIR-NIO under the project OLP2005.

Acknowledgments: The authors, C.R. and N.T., thank the Director, CSIR-NIO for the institutional support. This is the NIO's contribution number: 6741. Laurent Dufossé is indebted to the Conseil Régional de Bretagne, Conseil Régional de La Réunion, and the European Union for continuous support in the development of biotechnology research programs.

Conflicts of Interest: The authors declare no conflict of interest.

\section{References}

1. Shenkar, N.; Swalla, B.J. Global diversity of Ascidiacea. PLoS ONE 2011, 6, e20657. [CrossRef]

2. Holland, L.Z. Tunicates. Curr. Biol. 2016, 26, R141-R156. [CrossRef] [PubMed]

3. Gasparini, F.; Ballarin, L. Reproduction in Tunicates. In Encyclopedia of Reproduction, 2nd ed.; Elsevier Inc.: Amsterdam, The Netherlands, 2018; Volume 6, pp. 546-553.

4. Bone, Q.; Carré, C.; Chang, P. Tunicate feeding filters. J. Mar. Biol. Assoc. UK 2003, 83, 907-919. [CrossRef]

5. Delsuc, F.; Philippe, H.; Tsagkogeorga, G.; Simion, P.; Tilak, M.K.; Turon, X.; López-Legentil, S.; Piette, J.; Lemaire, P.; Douzery, E.J.P. A phylogenomic framework and timescale for comparative studies of tunicates. BMC Biol. 2018, 16, 39. [CrossRef] [PubMed]

6. Berna, L.; Alvarez-Valin, F. Evolutionary genomics of fast evolving tunicates. Genome Biol. Evol. 2014, 6, 1724-1738. [CrossRef]

7. Swalla, B.J. Building divergent body plans with similar genetic pathways. Heredity 2006, 97, 235-243. [CrossRef] [PubMed]

8. Jeffery, W.R. Tunicates: Models for Chordate Evolution and Development at Low Genomic Complexity. In Comparative Genomics; Clark, M.S., Ed.; Springer Science + Business Media: New York, NY, USA, 2000; pp. 43-69.

9. Lemaire, P. Evolutionary crossroads in developmental biology: The tunicates. Development 2011, 138, 2143-2152. [CrossRef]

10. Zhan, A.; Briski, E.; Bock, D.G.; Ghabooli, S.; MacIsaac, H.J. Ascidians as models for studying invasion success. Mar. Biol. 2015, 162, 2449-2470. [CrossRef]

11. Watters, D.J. Ascidian toxins with potential for drug development. Mar. Drugs 2018, 16, 162. [CrossRef]

12. Gouiffes, D.; Juge, M.; Grimaud, N.; Welin, L.; Sauviat, M.P.; Barbin, Y.; Laurent, D.; Roussakis, C.; Henichart, J.P.; Verbist, J.F. Bistramide A, a new toxin from the urochordata Lissoclinum bistratum Sluiter: Isolation and preliminary characterization. Toxicon 1988, 26, 1129-1136. [CrossRef]

13. Oh, K.-S.; Kim, J.-S.; Heu, M.-S. Food Constituents of Edible Ascidians Halocynthia roretzi and Pyura michaelseni. Korean J. Food Sci. Technol. 1997, 29, 955-962.

14. Ali, A.J.H.; Tamilselvi, M. Ascidians in Coastal Water: A Comprehensive Inventory of Ascidian Fauna from the Indian Coast; Springer Nature: Cham, Switzerland, 2016; ISBN 9783319291185.

15. DeFilippo, J.; Beck, G. Tunicate Immunology. In Reference Module in Life Sciences; Elsevier: Amsterdam, The Netherlands, 2018; pp. 1-10.

16. Ayuningrum, D.; Liu, Y.; Riyanti; Sibero, M.T.; Kristiana, R.; Asagabaldan, M.A.; Wuisan, Z.G.; Trianto, A.; Radjasa, O.K.; Sabdono, A.; et al. Tunicate-associated bacteria show a great potential for the discovery of antimicrobial compounds. PLoS ONE 2019, 14, e0213797. [CrossRef]

17. Franchi, N.; Ballarin, L. Immunity in protochordates: The tunicate perspective. Front. Immunol. 2017, 8, 674. [CrossRef] [PubMed]

18. Walters, T.L.; Gibson, D.M.; Frischer, M.E. Cultivation of the marine pelagic tunicate Dlioletta gegenbauri (Uljanin 1884) for experimental studies. J. Vis. Exp. 2019, 150, e59832. [CrossRef]

19. Fusetani, N. Drugs from the Sea; Krager: Basel, Switzerland, 2000.

20. Michibara, H.; Uyama, T.; Ueki, T.; Kanamori, K. The mechanism of accumulation and reduction of vanadium by ascidians. In The Biology of Ascidians; Sawada, H., Yokosawa, H., Lambert, C.C., Eds.; Springer: Tokyo, Japan, 2001; pp. $363-373$.

21. Shen, G.Q.; Baker, B.J. Biosynthetic studies of the eudistomins in the tunicate Eudistoma olivaceum. Tetrahedron Lett. 1994, 35, 1141-1144. [CrossRef]

22. Le, V.H.; Inai, M.; Williams, R.M.; Kan, T. Ecteinascidins. A review of the chemistry, biology and clinical utility of potent tetrahydroisoquinoline antitumor antibiotics. Nat. Prod. Rep. 2015, 32, 328-347. [CrossRef]

23. Xu, Y.; Kersten, R.D.; Nam, S.J.; Lu, L.; Al-Suwailem, A.M.; Zheng, H.; Fenical, W.; Dorrestein, P.C.; Moore, B.S.; Qian, P.Y. Bacterial biosynthesis and maturation of the didemnin anti-cancer agents. J. Am. Chem. Soc. 2012, 134, 8625-8632. [CrossRef]

24. Dewapriya, P.; Khalil, Z.G.; Prasad, P.; Salim, A.A.; Cruz-Morales, P.; Marcellin, E.; Capon, R.J. Talaropeptides A-D: Structure and biosynthesis of extensively N-methylated linear peptides from an Australian marine tunicate-derived Talaromyces sp. Front. Chem. 2018, 6, 394. [CrossRef]

25. Dou, X.; Dong, B. Origins and bioactivities of natural compounds derived from marine ascidians and their symbionts. Mar. Drugs 2019, 17, 670. [CrossRef]

26. Casertano, M.; Menna, M.; Imperatore, C. The ascidian-derived metabolites with antimicrobial properties. Antibiotics 2020,9 , 510. [CrossRef] 
27. Palanisamy, S.K.; Rajendran, N.M.; Marino, A. Natural products diversity of marine ascidians (Tunicates; Ascidiacea) and successful drugs in clinical development. Nat. Prod. Bioprospect. 2017, 7, 1-111. [CrossRef]

28. Petersen, J.K.; Riisgard, H.U. Filtration capacity of the ascidian Ciona intestinalis and its grazing impact in a shallow fjord. Mar. Ecol. Prog. Ser. 1992, 88, 9-17. [CrossRef]

29. Morris, R.J.; Bone, Q.; Head, R.; Braconnot, J.C.; Nival, P. Role of salps in the flux of organic matter to the bottom of the Ligurian Sea. Mar. Biol. 1988, 97, 237-241. [CrossRef]

30. Pomeroy, L.R.; Deibel, D. Aggregation of organic matter by pelagic tunicates. Limnol. Oceanogr. 1980, 25, 643-652. [CrossRef]

31. Gorsky, G.; Da Silva, N.L.; Dallot, S.; Laval, P.; Braconnot, J.C.; Prieur, L. Midwater tunicates: Are they related to the permanent front of the Ligurian Sea (NW Mediterranean)? Mar. Ecol. Prog. Ser. 1991, 74, 195-204. [CrossRef]

32. Sutherland, K.R.; Madin, L.P.; Stocker, R. Filtration of submicrometer particles by pelagic tunicates. Proc. Natl. Acad. Sci. USA 2010, 107, 15129-15134. [CrossRef]

33. Hirose, E.; Nozawa, Y. Latitudinal difference in the species richness of photosymbiotic ascidians along the east coast of Taiwan. Zool. Stud. 2020, 59, e19. [CrossRef] [PubMed]

34. Davis, A.R. Alkaloids and ascidian chemical defense: Evidence for the ecological role of natural products from Eudistoma olivaceum. Mar. Biol. 1991, 111, 375-379. [CrossRef]

35. Zhao, Y.; Li, J. Excellent chemical and material cellulose from tunicates: Diversity in cellulose production yield and chemical and morphological structures from different tunicate species. Cellulose 2014, 21, 3427-3441. [CrossRef]

36. Menna, M.; Aiello, A. The Chemistry of Marine Tunicates. In Handbook of Marine Natural Products; Fattorusso, E., Gerwick, W.H., Taglialatela-Scafati, O., Eds.; Springer Science + Business Media B.V.: Berlin/Heidelberg, Germany, 2012; pp. $295-385$.

37. Franchi, N.; Ballarin, L. Cytotoxic cells of compound Ascidians. In Lessons in Immunity: From Single-Cell Organisms to Mammals; Ballarin, L., Cammarata, M., Eds.; Elsevier Inc.: London, UK, 2016; pp. 193-199.

38. Parrinello, N. Cytotoxic activity of tunicate hemocytes. In Invertebrate Immunology; Rinkevich, B., Müller, W.E.G., Eds.; Springer: Berlin/Heidelberg, Germany, 1996; pp. 190-217.

39. Liu, L.; Zheng, Y.-Y.; Shao, C.-L.; Wang, C.-Y. Metabolites from marine invertebrates and their symbiotic microorganisms: Molecular diversity discovery, mining, and application. Mar. Life Sci. Technol. 2019, 1, 60-94. [CrossRef]

40. Leal, M.C.; Sheridan, C.; Osinga, R.; Dionísio, G.; Rocha, R.; Silva, B.; Rosa, R.; Calado, C. Marine Microorganism-Invertebrate Assemblages: Perspectives to Solve the "Supply Problem" in the Initial Steps of Drug Discovery. Mar. Drugs 2014, 12, 3929-3952. [CrossRef]

41. Chen, L.; Hu, J.S.; Xu, J.L.; Shao, C.L.; Wang, G.Y. Biological and chemical diversity of ascidian-associated microorganisms. Mar Drugs 2018, 16, 362. [CrossRef] [PubMed]

42. Oltz, E.M.; Smith, M.J.; Nakanishi, K.; Bruening, R.C.; Kustin, K. The tunichromes. A class of reducing blood pigments from sea squirts: Isolation, structures, and vanadium chemistry. J. Am. Chem. Soc. 1988, 110, 6162-6172. [CrossRef] [PubMed]

43. Kustin, K.; Robinson, W.E.; Smith, M.J. Tunichromes, vanadium, and vacuolated blood cells in tunicates. Invertebr. Reprod. Dev. 1990, 17, 129-139. [CrossRef]

44. Lee, I.H.; Zhao, C.; Cho, Y.; Harwig, S.S.L.; Cooper, E.L.; Lehrer, R.I. Clavanins, $\alpha$-helical antimicrobial peptides from tunicate hemocytes. FEBS Lett. 1997, 400, 158-162. [CrossRef]

45. Cima, F.; Franchi, N.; Ballarin, L. Origin and functions of tunicate hemocytes. In The Evolution of the Immune System: Conservation and Diversification; Malagoli, D., Ed.; Academic Press: London, UK, 2016; pp. 29-49. ISBN 9780128020135.

46. Galinier, R.; Roger, E.; Sautiere, P.E.; Aumelas, A.; Banaigs, B.; Mitta, G. Halocyntin and papillosin, two new antimicrobial peptides isolated from hemocytes of the solitary tunicate, Halocynthia papillosa. J. Pept. Sci. 2009, 15, 48-55. [CrossRef] [PubMed]

47. Jang, W.S.; Kim, K.N.; Lee, Y.S.; Nam, M.H.; Lee, I.H. Halocidin: A new antimicrobial peptide from hemocytes of the solitary tunicate, Halocynthia aurantium. FEBS Lett. 2002, 521, 81-86. [CrossRef]

48. Gorbman, A.; Whiteley, A.; Kavanaugh, S. Pheromonal stimulation of spawning release of gametes by gonadotropin releasing hormone in the chiton, Mopalia sp. Gen. Comp. Endocrinol. 2003, 131, 62-65. [CrossRef]

49. Evans, J.S.; Erwin, P.M.; Shenkar, N.; López-Legentil, S. Introduced ascidians harbor highly diverse and host-specific symbiotic microbial assemblages. Sci. Rep. 2017, 7, 11033. [CrossRef]

50. Tianero, M.D.B.; Kwan, J.C.; Wyche, T.P.; Presson, A.P.; Koch, M.; Barrows, L.R.; Bugni, T.S.; Schmidt, E.W. Species specificity of symbiosis and secondary metabolism in ascidians. ISME J. 2015, 9, 615-628. [CrossRef]

51. Flood, P. Yellow-stained oikopleurid appendicularians are caused by bacterial parasitism. Mar. Ecol. Prog. Ser. 1991, 71, 291-295. [CrossRef]

52. Cuadrado, A.; García-Fernández, L.F.; González, L.; Suárez, Y.; Losada, A.; Alcaide, V.; Martínez, T.; Máa Fernández-Sousa, J.; Sánchez-Puelles, J.M.; Muñoz, A. Aplidin ${ }^{\mathrm{TM}}$ induces apoptosis in human cancer cells via glutathione depletion and sustained activation of the epidermal growth factor receptor, Src, JNK, and p38 MAPK. J. Biol. Chem. 2003, 278, 241-250. [CrossRef] [PubMed]

53. Mitsiades, C.S.; Ocio, E.M.; Pandiella, A.; Maiso, P.; Gajate, C.; Garayoa, M.; Vilanova, D.; Montero, J.C.; Mitsiades, N.; McMullan, C.J.; et al. Aplidin, a marine organism-derived compound with potent antimyeloma activity in vitro and in vivo. Cancer Res. 2008. [CrossRef]

54. Urdiales, J.L.; Morata, P.; De Castro, I.N.; Sánchez-Jiménez, F. Antiproliferative effect of dehydrodidemnin B (DDB), a depsipeptide isolated from Mediterranean tunicates. Cancer Lett. 1996, 102, 31-37. [CrossRef] 
55. Fedorov, S.N.; Radchenko, O.S.; Shubina, L.K.; Balaneva, N.N.; Bode, A.M.; Stonik, V.A.; Dong, Z. Evaluation of cancer-preventive activity and structure- activity relationships of 3-demethylubiquinone Q2, isolated from the ascidian Aplidium glabrum, and it synthetic analogs. Pharm. Res. 2006, 23, 70-81. [CrossRef] [PubMed]

56. Garrido, L.; Zubía, E.; Ortega, M.J.; Salvá, J. Haouamines A and B: A new class of alkaloids from the ascidian Aplidium haouarianum. J. Org. Chem. 2003, 68, 293-299. [CrossRef] [PubMed]

57. Park, N.S.; Park, Y.K.; Ramalingam, M.; Yadav, A.K.; Cho, H.R.; Hong, V.S.; More, K.N.; Bae, J.H.; Bishop-Bailey, D.; Kano, J.; et al. Meridianin C inhibits the growth of YD-10B human tongue cancer cells through macropinocytosis and the down-regulation of Dickkopf-related protein-3. J. Cell. Mol. Med. 2018, 22, 5833-5846. [CrossRef]

58. Núñez-Pons, L.; Carbone, M.; Vázquez, J.; Rodríguez, J.; Nieto, R.M.; Varela, M.M.; Gavagnin, M.; Avila, C. Natural products from antarctic colonial ascidians of the genera Aplidium and Synoicum: Variability and defensive role. Mar. Drugs 2012, 10, 1741-1764. [CrossRef]

59. Paul, V.J.; Lindquist, N.; Fenical, W. Chemical defenses of the tropical ascidian Atapozoa sp. and its nudibranch predators Nembrotha spp. Mar. Ecol. Prog. Ser. 1990, 59, 109-118. [CrossRef]

60. Choi, H.; Hwang, H.; Chin, J.; Kim, E.; Lee, J.; Nam, S.J.; Lee, B.C.; Rho, B.J.; Kang, H. Tuberatolides, potent FXR antagonists from the korean marine tunicate Botryllus tuberatus. J. Nat. Prod. 2011, 74, 90-94. [CrossRef]

61. Kubanek, J.; Williams, D.E.; de Silva, E.D.; Allen, T.; Andersen, R.J. Cytotoxic alkaloids from the flatworm Prostheceraeus villatus and its tunicate prey Clavelina lepadiformis. Tetrahedron Lett. 1995, 36, 6189-6192. [CrossRef]

62. Raub, M.F.; Cardellina, J.H.; Choudhary, M.I.; Ni, C.Z.; Clardy, J.; Alley, M.C. Clavepictines A and B: Cytotoxic Quinolizidines from the Tunicate Clavelina picta. J. Am. Chem. Soc. 1991, 113, 3178-3180. [CrossRef]

63. Abourriche, A.; Abboud, Y.; Maoufoud, S.; Mohou, H.; Seffaj, T.; Charrouf, M.; Chaib, N.; Bennamara, A.; Bontemps, N.; Francisco, C. Cynthichlorine: A bioactive alkaloid from the tunicate Cynthia savignyi. Farmaco 2003, 58, 1351-1354. [CrossRef]

64. Kobayashi, J.; Cheng, J.F.; Nakamura, H.; Ohizumi, Y.; Walchli, M.R.; Hirata, Y.; Sasaki, T. Cystodytins A, B, and C, novel tetracyclic aromatic alkaloids with potent antineoplastic activity from the Okinawan tunicate Cystodytes dellechiajei. J. Org. Chem. 1988, 53, 1800-1804. [CrossRef]

65. Kobayashi, J.; Tsuda, M.; Tanabe, A.; Ishibashi, M.; Cheng, J.F.; Yamamura, S.; Sasaki, T. Cystodytins D-I, new cytotoxic tetracyclic aromatic alkaloids from the okinawan marine tunicate Cystodytes dellechiajei. J. Nat. Prod. 1991, 54, 1634-1638. [CrossRef] [PubMed]

66. Dassonneville, L.; Wattez, N.; Baldeyrou, B.; Mahieu, C.; Lansiaux, A.; Banaigs, B.; Bonnard, I.; Bailly, C. Inhibition of topoisomerase II by the marine alkaloid ascididemin and induction of apoptosis in leukemia cells. Biochem. Pharmacol. 2000, 60, 527-537. [CrossRef]

67. López-Legentil, S.; Turon, X.; Schupp, P. Chemical and physical defenses against predators in Cystodytes (Ascidiacea). J. Exp. Mar. Biol. Ecol. 2006, 332, 27-36. [CrossRef]

68. Uchimasu, H.; Matsumura, K.; Tsuda, M.; Kumagai, K.; Akakabe, M.; Fujita, M.J.; Sakai, R. Mellpaladines and dopargimine, novel neuroactive guanidine alkaloids from a Palauan Didemnidae tunicate. Tetrahedron 2016, 72, 7185-7193. [CrossRef]

69. Torii, M.; Hitora, Y.; Kato, H.; Koyanagi, Y.; Kawahara, T.; Losung, F.; Mangindaan, R.E.P.; Tsukamoto, S. Siladenoserinols M-P, sulfonated serinol derivatives from a tunicate. Tetrahedron 2018, 74, 7516-7521. [CrossRef]

70. Machida, K.; Arai, D.; Katsumata, R.; Otsuka, S.; Yamashita, J.K.; Ye, T.; Tang, S.; Fusetani, N.; Nakao, Y. Sameuramide A, a new cyclic depsipeptide isolated from an ascidian of the family Didemnidae. Bioorg. Med. Chem. 2018, 26, 3852-3857. [CrossRef] [PubMed]

71. Wright, A.D.; Goclik, E.; König, G.M.; Kaminsky, R. Lepadins D-F: Antiplasmodial and antitrypanosomal decahydroquinoline derivatives from the tropical marine tunicate Didemnum sp. J. Med. Chem. 2002, 45, 3067-3072. [CrossRef] [PubMed]

72. Mitchell, S.S.; Rhodes, D.; Bushman, F.D.; Faulkner, D.J. Cyclodidemniserinol trisulfate, a sulfated serinolipid from the Palauan ascidian Didemnum guttatum that inhibits HIV-1 integrase. Org. Lett. 2000, 2, 1605-1607. [CrossRef]

73. Seleghim, M.H.R.; De Lira, S.P.; Campana, P.T.; Berlinck, R.G.S.; Custódio, M.R. Localization of granulatimide alkaloids in the tissues of the ascidian Didemnum granulatum. Mar. Biol. 2007, 150, 967-975. [CrossRef]

74. Smith, T.E.; Pond, C.D.; Pierce, E.; Harmer, Z.P.; Kwan, J.; Zachariah, M.M.; Harper, M.K.; Wyche, T.P.; Matainaho, T.K.; Bugni, T.S.; et al. Accessing chemical diversity from the uncultivated symbionts of small marine animals. Nat. Chem. Biol. 2018, 14, 179-185. [CrossRef]

75. Donia, M.S.; Wang, B.; Dunbar, D.C.; Desai, P.V.; Patny, A.; Avery, M.; Hamann, M.T. Mollamides B and C, cyclic hexapeptides from the indonesian tunicate Didemnum molle. J. Nat. Prod. 2008, 71, 941-945. [CrossRef] [PubMed]

76. Oku, N.; Matsunaga, S.; Fusetani, N. Shishijimicins A-C, novel enediyne antitumor antibiotics from the ascidian Didemnum proliferum. J. Am. Chem. Soc. 2003, 125, 2044-2045. [CrossRef]

77. Takeara, R.; Jimenez, P.C.; Wilke, D.V.; Moraes, M.O.d.; Pessoa, C.; Lopes, N.P.; Lopes, J.L.C.; Lotufo, T.M.d.C.; Costa-Lotufo, L.V. Antileukemic effects of Didemnum psammatodes (Tunicata: Ascidiacea) constituents. Comp. Biochem. Physiol. A Mol. Integr. Physiol. 2008, 151, 363-369. [CrossRef]

78. Bracegirdle, J.; Robertson, L.P.; Hume, P.A.; Page, M.J.; Sharrock, A.V.; Ackerley, D.F.; Carroll, A.R.; Keyzers, R.A. Lamellarin Sulfates from the Pacific Tunicate Didemnum ternerratum. J. Nat. Prod. 2019, 82, 2000-2008. [CrossRef]

79. Charyulu, G.A.; McKee, T.C.; Ireland, C.M. Diplamine, a cytotoxic polyaromatic alkaloid from the tunicate Diplosoma sp. Tetrahedron Lett. 1989, 30, 4201-4202. [CrossRef] 
80. Ogi, T.; Taira, J.; Margiastuti, P.; Ueda, K. Cytotoxic metabolites from the Okinawan ascidian Diplosoma virens. Molecules 2008, 13, 595-602. [CrossRef]

81. Cvetkovic, R.S.; Figgitt, D.P.; Plosker, G.L. ET-743. Drugs 2002, 62, 1185-1192. [CrossRef]

82. Rashid, M.A.; Gustafson, K.R.; Boyd, M.R. New cytotoxic n-methylated $\beta$-carboline alkaloids from the marine ascidian Eudistoma gilboverde. J. Nat. Prod. 2001, 64, 1454-1456. [CrossRef] [PubMed]

83. Kobayashi, J.; Harbour, G.C.; Gilmore, J.; Rinehart, K.L. Eudistomins A, D, G, H, I, J, M, N, O, P, and Q, Bromo--, Hydroxy-, Pyrrolyl-, and 1-Pyrrolinyl- $\beta$-carbolines from the antiviral Caribbean tunicate Eudistoma olivaceum. J. Am. Chem. Soc. 1984, 106, 1526-1528. [CrossRef]

84. Rinehart, K.L.; Kobayashi, J.; Harbour, G.C.; Hughes, R.G.; Mizsak, S.A.; Scahill, T.A. Eudistomins C, E, K, and L, potent antiviral compounds containing a novel oxathiazepine ring from the Caribbean tunicate Eudistoma olivaceum. J. Am. Chem. Soc. 1984, 106, 1524-1526. [CrossRef]

85. Jimenez, P.C.; Wilke, D.V.; Ferreira, E.G.; Takeara, R.; De Moraes, M.O.; Silveira, E.R.; Lotufo, T.M.D.C.; Lopes, N.P.; Costa-Lotufo, L.V. Structure elucidation and anticancer activity of 7-oxostaurosporine derivatives from the Brazilian endemic tunicate Eudistoma vannamei. Mar. Drugs 2012, 10, 1092-1102. [CrossRef]

86. Rajesh, R.P.; Annappan, M. Anticancer effects of brominated indole alkaloid eudistomin H from marine ascidian Eudistoma viride against cervical cancer cells (HeLa). Anticancer Res. 2015, 35, 283-294.

87. Tapiolas, D.M.; Bowden, B.F.; Abou-Mansour, E.; Willis, R.H.; Doyle, J.R.; Muirhead, A.N.; Liptrot, C.; Llewellyn, L.E.; Wolff, C.W.W.; Wright, A.D.; et al. Eusynstyelamides A, B, and C, nNOS inhibitors, from the ascidian Eusynstyela latericius. J. Nat. Prod. 2009, 72, 1115-1120. [CrossRef]

88. Devi, S.; Rajasekharan, K.; Padmakumar, K.; Tanaka, J.; Higa, T. Biological activity and chemistry of the compound ascidian Eusynstyela tineta. In The Biology of Ascidians; Sawada, H., Yokosawa, H., Lambert, C.C., Eds.; Springer: Tokyo, Japan, 2001; pp. 341-354.

89. Tsukamotol, S.; Kato, H.; Hirota, H.; Fusetane, N. Lumichrome Is a putative intrinsic substance inducing larval metamorphosis in the ascidian Halocynthia roretzi. In The Biology of Ascidians; Sawada, H., Yokosawa, H., Lambert, C.C., Eds.; Springer: Tokyo, Japan, 2001; pp. 335-340.

90. Azumi, K.; Yokosawa, H.; Ishii, S.I. Halocyamines: Novel antimicrobial tetrapeptide-like substances isolated from the hemocytes of the solitary ascidian Halocynthia roretzi. Biochemistry 1990, 29, 159-165. [CrossRef]

91. Oda, T.; Fujiwara, T.; Liu, H.; Ukai, K.; Mangindaan, R.E.P.; Mochizuki, M.; Namikoshi, M. Effects of lissoclibadins and lissoclinotoxins, isolated from a tropical ascidian Lissoclinum cf. badium, on IL-8 production in a PMA-stimulated promyelocytic leukemia cell line. Mar. Drugs 2006, 4, 15-21. [CrossRef]

92. Kumaran, N.S.; Bragadeeswaran, S.; Meenakshi, V.K.; Balasubramanian, T. Bioactivity potential of extracts from ascidian Lissoclinum fragile. Afr. J. Pharm. Pharmacol. 2012, 6, 1854-1859. [CrossRef]

93. Corley, D.G.; Moore, R.E.; Paul, V.J. Patellazole B: A novel cytotoxic thiazole-containing macrolide from the marine tunicate Lissoclinum patella. J. Am. Chem. Soc. 1988, 110, 7920-7922. [CrossRef]

94. Zabriskie, T.M.; Mayne, C.L.; Ireland, C.M. Patellazole C: A novel cytotoxic macrolide from Lissoclinum patella. J. Am. Chem. Soc. 1988, 110, 7919-7920. [CrossRef]

95. Odate, S.; Pawlik, J.R. The role of vanadium in the chemical defense of the solitary tunicate, Phallusia nigra. J. Chem. Ecol. 2007, 33, 643-654. [CrossRef]

96. Casertano, M.; Imperatore, C.; Luciano, P.; Aiello, A.; Putra, M.Y.; Gimmelli, R.; Ruberti, G.; Menna, M. Chemical investigation of the indonesian tunicate Polycarpa aurata and evaluation of the effects against Schistosoma mansoni of the novel alkaloids polyaurines A and B. Mar. Drugs 2019, 17, 278. [CrossRef]

97. Kang, H.; Fenical, W. Polycarpine dihydrochloride: A cytotoxic dimeric disulfide alkaloid from the Indian ocean ascidian Polycarpa clavata. Tetrahedron Lett. 1996, 37, 2369-2372. [CrossRef]

98. Guo, P.; Wang, Z.; Li, G.; Liu, Y.; Xie, Y.; Wang, Q. First discovery of polycarpine, polycarpaurines A and C, and their derivatives as novel antiviral and antiphytopathogenic fungus agents. J. Agric. Food Chem. 2016, 64, 4264-4272. [CrossRef]

99. Fujita, M.; Nakao, Y.; Matsunaga, S.; Nishikawa, T.; Fusetani, N. Sodium 1-(12-hydroxy)octadecanyl sulfate, an MMP2 inhibitor, isolated from a tunicate of the family polyclinidae. J. Nat. Prod. 2002, 65, 1936-1938. [CrossRef]

100. Nicolaou, K.C.; Li, R.; Lu, Z.; Pitsinos, E.N.; Alemany, L.B. Total synthesis and full structural assignment of namenamicin. J. Am. Chem. Soc. 2018, 140, 8091-8095. [CrossRef]

101. Cheng, M.T.; Rinehart, K.L. Polyandrocarpidines: Antimicrobial and Cytotoxic Agents from a Marine Tunicate (Polyandrocarpa sp.) from the Gulf of California. J. Am. Chem. Soc. 1978, 100, 7409-7411. [CrossRef]

102. Lindquist, N.; Hay, M.E.; Fenical, W. Defense of ascidians and their conspicuous larvae: Adult vs. larval chemical defenses. Ecol. Monogr. 1992, 62, 547-568. [CrossRef]

103. Kaneko, N.; Katsuyama, Y.; Kawamura, K.; Fujiwara, S. Regeneration of the gut requires retinoic acid in the budding ascidian Polyandrocarpa misakiensis. Dev. Growth Differ. 2010, 52, 457-468. [CrossRef]

104. Wang, W.; Kim, H.; Nam, S.J.; Rho, B.J.; Kang, H. Antibacterial butenolides from the korean tunicate Pseudodistoma antinboja. J. Nat. Prod. 2012, 27, 574-577. [CrossRef] [PubMed]

105. Appleton, D.R.; Page, M.J.; Lambert, G.; Berridge, M.V.; Copp, B.R. Kottamides A-D: Novel bioactive imidazolone-containing alkaloids from the New Zealand ascidian Pycnoclavella kottae. J. Org. Chem. 2002, 67, 5402-5404. [CrossRef] 
106. Aiello, A.; Carbonelli, S.; Fattorusso, E.; Iuvone, T.; Menna, M. New bioactive sulfated metabolites from the Mediterranean tunicate Sidnyum turbinatum. J. Nat. Prod. 2001, 64, 219-221. [CrossRef]

107. Davies-Coleman, M.T.; Cantrell, C.L.; Gustafson, K.R.; Beutler, J.A.; Pannell, L.K.; Boyd, M.R. Stolonic acids A and B, new cytotoxic cyclic peroxides from an Indian Ocean ascidian Stolonica species. J. Nat. Prod. 2000, 63, 1411-1413. [CrossRef]

108. Menzel, L.P.; Lee, I.H.; Sjostrand, B.; Lehrer, R.I. Immunolocalization of clavanins in Styela clava hemocytes. Dev. Comp. Immunol. 2002, 26, 505-515. [CrossRef]

109. Raftos, D.A.; Hutchinson, A. Cytotoxicity reactions in the solitary tunicate Styela plicata. Dev. Comp. Immunol. 1995, $19,463-471$. [CrossRef]

110. Miyata, Y. Ecdysteroids from the Antarctic tunicate Synoicum adareanum. J. Nat. Prod. 2007, 70, 1859-1864. [CrossRef]

111. Rinehart, K.L.; Gloer, J.B.; Hughes, R.G.; Renis, H.E.; Patrick McGovren, J.; Swynenberg, E.B.; Stringfellow, D.A.; Kuentzel, S.L.; Li, L.H. Didemnins: Antiviral and antitumor depsipeptides from a Caribbean tunicate. Science 1981, 212, 933-935. [CrossRef]

112. Rodríguez-Martínez, R.E.; Jordán-Garza, A.G.; Baker, D.M.; Jordán-Dahlgren, E. Competitive interactions between corals and Trididemnum solidum on Mexican Caribbean reefs. Coral Reefs 2012, 31, 571-577. [CrossRef]

113. Schofield, M.M.; Jain, S.; Porat, D.; Dick, G.J.; Sherman, D.H. Identification and analysis of the bacterial endosymbiont specialized for production of the chemotherapeutic natural product ET-743. Environ. Microbiol. 2015, 17, 3964-3975. [CrossRef] [PubMed]

114. Jayanetti, D.R.; Braun, D.R.; Barns, K.J.; Rajski, S.R.; Bugni, T.S. Bulbiferates A and B: Antibacterial acetamidohydroxybenzoates from a marine proteobacterium, Microbulbifer sp. J. Nat. Prod. 2019, 82, 1930-1934. [CrossRef] [PubMed]

115. Yamazaki, H.; Nakayama, W.; Takahashi, O.; Kirikoshi, R.; Izumikawa, Y.; Iwasaki, K.; Toraiwa, K.; Ukai, K.; Rotinsulu, H.; Wewengkang, D.S.; et al. Verruculides A and B, two new protein tyrosine phosphatase 1B inhibitors from an Indonesian ascidian-derived Penicillium verruculosum. Bioorganic Med. Chem. Lett. 2015, 25, 3087-3090. [CrossRef]

116. Pinkerton, D.M.; Banwell, M.G.; Garson, M.J.; Kumar, N.; De Moraes, M.O.; Cavalcanti, B.C.; Barros, F.W.A.; Pessoa, C. Antimicrobial and cytotoxic activities of synthetically derived tambjamines C and E-J, BE-18591, and a related alkaloid from the marine bacterium Pseudoalteromonas tunicata. Chem. Biodivers. 2010, 7, 1311-1324. [CrossRef]

117. Burke, C.; Thomas, T.; Egan, S.; Kjelleberg, S. The use of functional genomics for the identification of a gene cluster encoding for the biosynthesis of an antifungal tambjamine in the marine bacterium Pseudoalteromonas tunicata: Brief report. Environ. Microbiol. 2007, 9, 814-818. [CrossRef]

118. Wang, K.L.; Xu, Y.; Lu, L.; Li, Y.; Han, Z.; Zhang, J.; Shao, C.L.; Wang, C.Y.; Qian, P.Y. Low-toxicity diindol-3-ylmethanes as potent antifouling compounds. Mar. Biotechnol. 2015, 17, 624-632. [CrossRef] [PubMed]

119. Takagi, M.; Motohashi, K.; Izumikawa, M.; Khan, S.T.; Hwang, J.-H.; Shin-Ya, K. JBIR-66, a new metabolite isolated from tunicate-derived Saccharopolyspora sp. SS081219JE-28. Biosci. Biotechnol. Biochem. 2010, 74, 2355-2357. [CrossRef]

120. Wasserman, H.H.; Friedland, D.J.; Morrison, D.A. A novel dipyrrolyldipyrromethene prodigiosin analog from Serratia marcescens. Tetrahedron Lett. 1968, 6, 641-644. [CrossRef]

121. Sung, A.A.; Gromek, S.M.; Balunas, M.J. Upregulation and identification of antibiotic activity of a marine-derived Streptomyces sp. via co-cultures with human pathogens. Mar. Drugs 2017, 15, 250. [CrossRef] [PubMed]

122. Tsukimoto, M.; Nagaoka, M.; Shishido, Y.; Fujimoto, J.; Nishisaka, F.; Matsumoto, S.; Harunari, E.; Imada, C.; Matsuzaki, T. Bacterial production of the tunicate-derived antitumor cyclic depsipeptide didemnin B. J. Nat. Prod. 2011, 74, 2329-2331. [CrossRef]

123. Karthikeyan, M.M.; Ananthan, G.; Balasubramanian, T. Antimicrobial activity of crude extracts of some ascidians (Urochordata: Ascidiacea), from Palk Strait, (Southeast Coast of India). World J. Fish. Mar. Sci. 2009, 1, $262-267$.

124. Ayuningrum, D.; Kristiana, R.; Nisa, A.A.; Radjasa, S.K.; Muchlissin, S.I.; Radjasa, O.K.; Sabdono, A.; Trianto, A. Bacteria associated with tunicate, Polycarpa aurata, from Lease sea, Maluku, Indonesia exhibiting anti-multidrug resistant bacteria. Biodiversitas 2019, 20, 956-964. [CrossRef]

125. Litaay, M.; Christine, G.; Gobel, R.B.; Dwyana, Z. Bioactivity of endo-symbiont bacteria of tunicate Polycarpa aurata as antimicrobial. In Proceedings of the 23 National Seminar of Indonesia Biology Society, Jayapura, Indonesia, 18 September 2015.

126. Menna, M.; Fattorusso, E.; Imperatore, C. Alkaloids from marine ascidians. Molecules 2011, 16, 8694-8732. [CrossRef]

127. Franks, A.; Haywood, P.; Holmström, C.; Egan, S.; Kjelleberg, S.; Kumar, N. Isolation and structure elucidation of a novel yellow pigment from the marine bacterium Pseudoalteromonas tunicata. Molecules 2005, 10, 1286-1291. [CrossRef] [PubMed]

128. Franks, A.; Egan, S.; Holmström, C.; James, S.; Lappin-Scott, H.; Kjelleberg, S. Inhibition of fungal colonization by Pseudoalteromonas tunicata provides a competitive advantage during surface colonization. Appl. Environ. Microbiol. 2006, 72, 6079-6087. [CrossRef]

129. Egan, S.; James, S.; Holmström, C.; Kjelleberg, S. Correlation between pigmentation and antifouling compounds produced by Pseudoalteromonas tunicata. Environ. Microbiol. 2002, 4, 433-442. [CrossRef]

130. Matz, C.; Webb, J.S.; Schupp, P.J.; Phang, S.Y.; Penesyan, A.; Egan, S.; Steinberg, P.; Kjelleberg, S. Marine biofilm bacteria evade eukaryotic predation by targeted chemical defense. PLOS ONE 2008, 3, e2744. [CrossRef]

131. Holmström, C.; James, S.; Neilan, B.A.; White, D.C.; Kjelleberg, S. Pseudoalteromonas tunicata sp. nov., a bacterium that produces antifouling agents. Int. J. Syst. Bacteriol. 1998, 48, 1205-1212. [CrossRef]

132. James, S.G.; Holmström, C.; Kjelleberg, S. Purification and characterization of a novel antibacterial protein from the marine bacterium D2. Appl. Environ. Microbiol. 1996, 62, 2783-2788. [CrossRef] 
133. Fedders, H.; Michalek, M.; Grötzinger, J.; Leippe, M. An exceptional salt-tolerant antimicrobial peptide derived from a novel gene family of haemocytes of the marine invertebrate Ciona intestinalis. Biochem. J. 2008, 416, 65-75. [CrossRef] [PubMed]

134. Nurfadillah, A.; Litaay, M.; Gobel, R.B.; Haedar, N. Potency of tunicate Polycarpa aurata as inoculum source of sebagai sumber endosimbyotic fungi that produce antimicrobe. J. Alam Lingkung. 2015, 6, 10-16.

135. Tahir, E.; Litaay, M.; Gobel, R.B.; Haedar, N.; Al, E. Potency of tunicate Rhopalaea crassa as inoculum source of endosymbiont fungi that produce antimicrobe. Spermonde 2016, 2, 33-37.

136. Shaala, L.A.; Youssef, D.T.A. Identification and bioactivity of compounds from the fungus Penicillium sp. CYE-87 isolated from a marine tunicate. Mar. Drugs 2015, 13, 1698-1709. [CrossRef] [PubMed]

137. Canonico, P.G.; Pannier, W.L.; Huggins, J.W.; Rienehart, K.L. Inhibition of RNA viruses in vitro and in Rift Valley fever-infected mice by didemnins A and B. Antimicrob. Agents Chemother. 1982, 22, 696-697. [CrossRef] [PubMed]

138. Mayer, A.M.S.; Glaser, K.B.; Cuevas, C.; Jacobs, R.S.; Kem, W.; Little, R.D.; McIntosh, J.M.; Newman, D.J.; Potts, B.C.; Shuster, D.E. The odyssey of marine pharmaceuticals: A current pipeline perspective. Trends Pharmacol. Sci. 2010, 31, 255-265. [CrossRef]

139. Zelek, L.; Yovine, A.; Brain, E.; Turpin, F.; Taamma, A.; Riofrio, M.; Spielmann, M.; Jimeno, J.; Misset, J.L. A phase II study of Yondelis ${ }^{\circledR}$ (trabectedin, ET-743) as a 24-h continuous intravenous infusion in pretreated advanced breast cancer. Br. J. Cancer 2006, 94, 1610-1614. [CrossRef]

140. Atmaca, H.; Bozkurt, E.; Uzunoglu, S.; Uslu, R.; Karaca, B. A diverse induction of apoptosis by trabectedin in MCF-7 (HER2-/ER+) and MDA-MB-453 (HER2+/ER-) breast cancer cells. Toxicol. Lett. 2013, 221, 128-136. [CrossRef] [PubMed]

141. Grosso, F.; Jones, R.L.; Demetri, G.D.; Judson, I.R.; Blay, J.-Y.; Cesne, A.L.; Lippo, R.S.; Casieri, P.; Collini, P.; Dileo, P.; et al. Effi cacy of trabectedin (ecteinascidin-743) in advanced pretreated myxoid liposarcomas: A retrospective study. Lancet Oncol. 2007, 8, 595-602. [CrossRef]

142. Sessa, C.; De Braud, F.; Perotti, A.; Bauer, J.; Curigliano, G.; Noberasco, C.; Zanaboni, F.; Gianni, L.; Marsoni, S.; Jimeno, J.; et al. Trabectedin for women with ovarian carcinoma after treatment with platinum and taxanes fails. J. Clin. Oncol. 2005, 23, 1867-1874. [CrossRef]

143. Krasner, C.N.; McMeekin, D.S.; Chan, S.; Braly, P.S.; Renshaw, F.G.; Kaye, S.; Provencher, D.M.; Campos, S.; Gore, M.E. A Phase II study of trabectedin single agent in patients with recurrent ovarian cancer previously treated with platinum-based regimens. $\mathrm{Br}$. J. Cancer 2007, 97, 1618-1624. [CrossRef]

144. Monk, B. A randomized phase III study of trabectedin with pegylated liposomal doxorubicin (PLD) versus PLD in relapsed, recurrent ovarian cancer (OC). Eur. J. Cancer Suppl. 2008, 19, viii1-viii4.

145. Rath, C.M.; Janto, B.; Earl, J.; Ahmed, A.; Hu, F.Z.; Hiller, L.; Dahlgren, M.; Kreft, R.; Yu, F.; Wolff, J.J.; et al. Meta-omic characterization of the marine invertebrate microbial consortium that produces the chemotherapeutic natural product ET-743. ACS Chem. Biol. 2011, 6, 1244-1255. [CrossRef] [PubMed]

146. Tohme, R.; Darwiche, N.; Gali-Muhtasib, H. A journey under the sea: The quest for marine anti-cancer alkaloids. Molecules 2011, 16, 9665-9696. [CrossRef]

147. González-Santiago, L.; Suárez, Y.; Zarich, N.; Muñoz-Alonso, M.J.; Cuadrado, A.; Martínez, T.; Goya, L.; Iradi, A.; Sáez-Tormo, G.; Maier, J.V.; et al. Aplidin ${ }^{\circledR}$ induces JNK-dependent apoptosis in human breast cancer cells via alteration of glutathione homeostasis, Rac1 GTPase activation, and MKP-1 phosphatase downregulation. Cell Death Differ. 2006, 13, 1968-1981. [CrossRef] [PubMed]

148. Rinehart, K.L. Antitumor compounds from tunicates. Med. Res. Rev. 2000, 20, 1-27. [CrossRef]

149. Kobayashi, J.; Cheng, J.F.; Nakamura, H.; Ohta, T.; Nozoe, S.; Hirata, Y.; Sasaki, T. Lejimalides A and B, novel 24-membered macrolides with potent antileukemic activity from the Okinawan tunicate Eudistoma cf. rigida. J. Org. Chem. 1988, 53, 6147-6150. [CrossRef]

150. Kikuchi, Y.; Ishibashi, M.; Sasaki, T.; Kobayashi, J. Lejimalides C and D, new antineoplastic 24-membered macrolide sulfates from the okinawan marine tunicate Eudistoma cf. rigida. Tetrahedron Lett. 1991, 32, 789-797. [CrossRef]

151. Nguyen, M.H.; Imanishi, M.; Kurogi, T.; Wan, X.; Ishmael, J.E.; McPhail, K.L.; Smith, A.B. Synthetic access to the mandelalide family of macrolides: Development of an anion relay chemistry strategy. J. Org. Chem. 2018, 83, 4287-4306. [CrossRef]

152. Rinehart, K.L.; Holt, T.G.; Fregeau, N.L.; Stroh, J.G.; Keifer, P.A.; Sun, F.; Li, L.H.; Martin, D.G. Ecteinascidins 729, 743, 745, 759A, 759B, and 770: Potent antitumor agents from the Caribbean tunicate Ecteinascidia turbinata. J. Org. Chem. 1990, 55, 4512-4515. [CrossRef]

153. Izbicka, E.; Lawrence, R.; Raymond, E.; Eckhardt, G.; Faircloth, G.; Jimeno, J.; Clark, G.; Von Hoff, D.D. In vitro antitumor activity of the novel marine agent, Ecteinascidin-743 (ET-743, NSC-648766) against human tumors explanted from patients. Ann. Oncol. 1998, 9, 981-987. [CrossRef]

154. Berlmck, R.G.S.; Britton, R.; Piers, E.; Lim, L.; Roberge, M.; Moreira Da Rocha, R.; Andersen, R.J. Granulatimide and isogranulatimide, aromatic alkaloids with G2 checkpoint inhibition activity isolated from the Brazilian ascidian Didemnum granulatum: Structure elucidation and synthesis. J. Org. Chem. 1998, 63, 9850-9856. [CrossRef]

155. Holmstrom, C.; James, S.; Egan, S.; Kjelleberg, S. Inhibition of common fouling organisms by marine bacterial isolates with special reference to the role of pigmented bacteria. Biofouling 1996, 10, 251-259. [CrossRef]

156. Holmstrom, C.; Rittschof, D.; Kjelleberg, S. Inhibition of settlement by larvae of Balanus amphitrite and Ciona intestinalis by a surface-colonizing marine bacterium. Appl. Environ. Microbiol. 1992, 58, 2111-2115. [CrossRef] [PubMed]

157. Stoecker, D. Resistance of a tunicate to fouling. Biol. Bull. 1978, 155, 615-626. [CrossRef] 
158. Vervoort, H.C.; Pawlik, J.R.; Fenical, W. Chemical defense of the Caribbean ascidian Didemnum conchyliatum. Mar. Ecol. Prog. Ser. 1998, 164, 221-228. [CrossRef]

159. Lindquist, N.; Hay, M.E. Can small rare prey be chemically defended? The case for marine larvae. Ecology 1995, 76, 1347-1358. [CrossRef]

160. Lindquist, N.; Fenical, W. New tambjamine class alkaloids from the marine ascidian Atapozoa sp. and its nudibranch predators. Origin of the tambjamines in Atapozoa. Experientia 1991, 47, 504-506. [CrossRef]

161. McClintock, J.B.; Amsler, M.O.; Koplovitz, G.; Amsler, C.D.; Baker, B.J. Observations on an association between the dexaminid amphipod Polycheria antarctica f. acanthopoda and its ascidian host Distaplia cylindrica. J. Crustac. Biol. 2009, 29, 605-608. [CrossRef]

162. Watanabe, T.; Shibata, H.; Ebine, M.; Tsuchikawa, H.; Matsumori, N.; Murata, M.; Yoshida, M.; Morisawa, M.; Lin, S.; Yamauchi, K.; et al. Synthesis and complete structure determination of a sperm-activating and -attracting factor isolated from the ascidian ascidia sydneiensis. J. Nat. Prod. 2018, 81, 985-997. [CrossRef]

163. Mikami, N.; Hosokawa, M.; Miyashita, K. Effects of sea squirt (Halocynthia roretzi) lipids on white adipose tissue weight and blood glucose in diabetic/obese KK-Ay mice. Mol. Med. Rep. 2010, 3, 449-453. [PubMed]

164. Thakur, N.L. Studies on Some Bioactive Aspects of Selected Marine Organisms; Goa University: Goa, India, 2001.

165. Kazlauskas, R.; Marwood, J.F.; Murphy, P.T.; Wells, R.J. A blue pigment from a compound ascidian. Aust. J. Chem. 1982, 35, 215-217. [CrossRef]

166. Wright, A.E.; Forleo, D.A.; Gunawardana, G.P.; Gunasekera, S.P.; Koehn, F.E.; McConnell, O.J. Antitumor tetrahydroisoquinoline alkaloids from the colonial ascidian Ecteinascidia turbinata. J. Org. Chem. 1990, 55, 4508-4515. [CrossRef]

167. Kott, P. Didemnid-algal symbiosis: Host species in the Western Pacific with notes on the symbiosis. Micronesica 1982, 18, 95-127.

168. Sings, H.L.; Rinehart, K.L. Compounds produced from potential tunicate-blue-green algal symbiosis: A review. J. Ind. Microbiol. Biotechnol. 1996, 17, 385-396. [CrossRef]

169. Carté, B.; Faulkner, D.J. Defensive metabolites from three nembrothid nudibranchs. J. Org. Chem. 1983, 48, 2314-2318. [CrossRef]

170. Garson, M.J. Marine natural products as antifeedants. In Comprehensive Natural Products II: Chemistry and Biology; Elsevier Ltd.: Amsterdam, The Netherlands, 2010; pp. 503-537. ISBN 9780080453828.

171. Lins, D.M.; de Marco, P.; Andrade, A.F.A.; Rocha, R.M. Predicting global ascidian invasions. Divers. Distrib. 2018, $24,692-704$. [CrossRef]

172. Gittenberger, A.; Moons, J.J.S. Settlement and possible competition for space between the invasive violet tunicate Botrylloides violaceus and the native star tunicate Botryllus schlosseri in The Netherlands. Aquat. Invasions 2011, 6, 435-440. [CrossRef]

173. Sephton, D.; Vercaemer, B.; Nicolas, J.M.; Keays, J. Monitoring for invasive tunicates in Nova Scotia, Canada (2006-2009). Aquat. Invasions 2011, 6, 391-403. [CrossRef]

174. Seo, K.S.; Lee, Y. A first assessment of invasive marine species on Chinese and Korean coasts. In Biological Invasions in Marine Ecosystems, Ecological Studies 204; Rilov, G., Crooks, J.A., Eds.; Springer: Berlin/Heidelberg, Germany, 2009 ; pp. 577-585.

175. Costello, K.E.; Lynch, S.A.; McAllen, R.; O’Riordan, R.M.; Culloty, S.C. The role of invasive tunicates as reservoirs of molluscan pathogens. Biol. Invasions 2021, 23, 641-655. [CrossRef]

176. Daigle, R.M.; Herbinger, C.M. Ecological interactions between the vase tunicate (Ciona intestinalis) and the farmed blue mussel (Mytilus edulis) in Nova Scotia, Canada. Aquat. Invasions 2009, 4, 177-187. [CrossRef]

177. Ramesh, C.H.; Koushik, S.; Shunmugaraj, T.; Murthy, M.V.R. Infestation of colonial ascidians on reef biota of Gulf of Mannar Marine Biosphere Reserve, India. J. New Biol. Rep. 2019, 8, 187-189.

178. Vargas-Ángel, B.; Godwin, L.S.; Asher, J.; Brainard, R.E. Invasive didemnid tunicate spreading across coral reefs at remote Swains Island, American Sāmoa. Coral Reefs 2009, 28, 53. [CrossRef]

179. Tatián, M.; Schwindt, E.; Lagger, C.; Varela, M.M. Colonization of Patagonian harbours (SW Atlantic) by an invasive sea squirt. Spixiana 2010, 33, 111-117.

180. Pleus, A.; LeClair, L.; Schultz, J.; Lambert, G. 2007-09 Tunicate Management Plan; In Coordination with the Tunicate Response Advisory Committee; Washington State Department of Fish and Wildlife, Aquatic Invasive Species Unit: Olympia, WA, USA, 2008; pp. 1-64.

181. Cordell, J.R.; Levy, C.; Toft, J.D. Ecological implications of invasive tunicates associated with artificial structures in Puget Sound, Washington, USA. Biol. Invasions 2013, 15, 1303-1318. [CrossRef]

182. Abdul Jaffar, H.; Soban Akram, A.; Kaleem Arshan, M.L.; Sivakumar, V.; Tamilselvi, M. Distribution and invasiveness of a colonial ascidian, Didemnum psammathodes, along the southern Indian coastal water. Oceanologia 2016, 58, 212-220. [CrossRef]

183. Carman, M.R.; Grunden, D.W. First occurrence of the invasive tunicate Didemnum vexillum in eelgrass habitat. Aquat. Invasions 2010, 5, 23-29. [CrossRef]

184. Griffith, K.; Mowat, S.; Holt, R.H.F.; Ramsay, K.; Bishop, J.D.D.; Lambert, G.; Jenkins, S.R. First records in Great Britain of the invasive colonial ascidian Didemnum vexillum Kott, 2002. Aquat. Invasions 2009, 4, 581-590. [CrossRef]

185. Sorte, C.J.B.; Williams, S.L.; Zerebecki, R.A. Ocean warming increases threat of invasive species in a marine fouling community. Ecology 2010, 91, 2198-2204. [CrossRef] 